ARTICLE

\title{
TGF $\beta$ promotes fibrosis by MYST1-dependent epigenetic regulation of autophagy
}

\author{
Ariella Zehender ${ }^{1,2,8}$, Yi-Nan Li (10) 1,2,8, Neng-Yu Lin 1,2,3,8, Adrian Stefanica1,2, Julian Nüchel (1) 4, \\ Chih-Wei Chen 1,2, Hsiao-Han Hsu ${ }^{3}$, Honglin Zhu1,2,5, Xiao Ding ${ }^{1,2}$, Jingang Huang ${ }^{1,2}$, Lichong Shen ${ }^{1,2}$, \\ Andrea-Hermina Györfi (10) 1,2, Alina Soare 1,2, Simon Rauber (10 1,2, Christina Bergmann 1,2, Andreas Ramming 1,2,

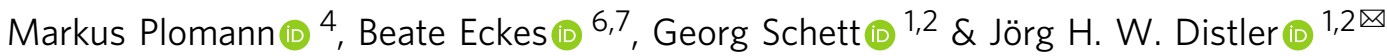

Activation of fibroblasts is essential for physiological tissue repair. Uncontrolled activation of fibroblasts, however, may lead to tissue fibrosis with organ dysfunction. Although several pathways capable of promoting fibroblast activation and tissue repair have been identified, their interplay in the context of chronic fibrotic diseases remains incompletely understood. Here, we provide evidence that transforming growth factor- $\beta$ (TGF $\beta$ ) activates autophagy by an epigenetic mechanism to amplify its profibrotic effects. TGF $\beta$ induces autophagy in fibrotic diseases by SMAD3-dependent downregulation of the H4K16 histone acetyltransferase MYST1, which regulates the expression of core components of the autophagy machinery such as ATG7 and BECLIN1. Activation of autophagy in fibroblasts promotes collagen release and is both, sufficient and required, to induce tissue fibrosis. Forced expression of MYST1 abrogates the stimulatory effects of TGF $\beta$ on autophagy and re-establishes the epigenetic control of autophagy in fibrotic conditions. Interference with the aberrant activation of autophagy inhibits TGF $\beta$-induced fibroblast activation and ameliorates experimental dermal and pulmonary fibrosis. These findings link uncontrolled TGF $\beta$ signaling to aberrant autophagy and deregulated epigenetics in fibrotic diseases and may contribute to the development of therapeutic interventions in fibrotic diseases.

\footnotetext{
${ }^{1}$ Department of Internal Medicine 3-Rheumatology and Immunology, Friedrich-Alexander-University Erlangen-Nürnberg (FAU) and University Hospital Erlangen, Erlangen, Germany. ${ }^{2}$ Deutsches Zentrum für Immuntherapie, Friedrich Alexander University Erlangen-Nuremberg and Universitaetsklinikum Erlangen, Erlangen, Germany. ${ }^{3}$ Graduate Institute of Anatomy and Cell Biology, College of Medicine, National Taiwan University, Taipei, Taiwan. ${ }^{4}$ Center for Biochemistry, University of Cologne, Faculty of Medicine, Cologne, Germany. ${ }^{5}$ Department of Rheumatology, Xiangya Hospital, Central South University, Changsha, Hunan, China. ${ }^{6}$ Translational Matrix Biology, University of Cologne, Faculty of Medicine, Cologne, Germany. ${ }^{7}$ Cologne Excellence Cluster on Cellular Stress Responses in Aging-Associated Diseases (CECAD), Cologne, Germany. ${ }^{8}$ These authors contributed equally: Ariella Zehender, Yi-Nan Li,

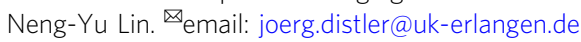


F brotic diseases are characterized by excessive deposition of extracellular matrix with perturbation of the physiological tissue architecture. Fibrotic tissue remodeling imposes a major burden on modern societies and has been estimated to contribute to up to $45 \%$ of deaths in the developed world ${ }^{1}$. In addition to high mortality, many fibrotic diseases are also associated with severe morbidity and often cause lifelong disability, which results in socioeconomic costs in the order of tens of billions of dollars per year ${ }^{2}$. Fibrotic tissue remodeling can occur in response to defined stimuli such as trauma, infection or tumors, whereas the initiating trigger remains unknown in other cases ${ }^{3}$. Those idiopathic fibrotic diseases can affect virtually every organ system of the human body. Systemic sclerosis (SSc) is a prototypical example of an idiopathic fibrotic disease with high mortality and morbidity ${ }^{4}$. SSc most commonly affects the skin and lungs, but may also involve other internal organs such as the heart and the intestinal tract. SSc and other fibrotic diseases are characterized by aberrant activation of resident fibroblasts that display a persistently activated myofibroblast phenotype. While myofibroblasts are only transiently observed during normal wound healing, they persist in fibrotic diseases, thereby leading to excessive repair responses that culminate in tissue fibrosis ${ }^{5}$.

Although the molecular mechanisms underlying the aberrant fibroblast activation in fibrotic tissues remain incompletely understood, overwhelming evidence highlights that transforming growth factor- $\beta$ (TGF $\beta$ ) can promote fibroblast activation and myofibroblast differentiation ${ }^{6}$. TGF $\beta$ signaling is upregulated in fibrotic diseases with persistent activation of intracellular downstream mediators and a TGF $\beta$ biased gene expression signature in affected tissues $^{7}$. Moreover, forced activation of TGF $\beta$ signaling induces a myofibroblast phenotype in resting fibroblasts and systemic fibrosis in mice ${ }^{8}$. Increased TGF $\beta$ signaling is not only sufficient, but also required for fibrosis as inhibition of TGF $\beta$ or its receptors ameliorates fibrosis in multiple experimental models ${ }^{6}$.

Epigenetic alterations are increasingly recognized as drivers of progression of fibrotic diseases 9,10 . The expression of several epigenetic regulators including DNA methyltransferases, noncoding RNAs, histone demethylases, and histone acetyltransferases is deregulated in fibrotic diseases ${ }^{10-12}$. The resulting changes in the epigenetic code skew the transcriptome towards a profibrotic gene expression profile, thereby enabling effector cells such as fibroblasts to maintain their activated phenotype even in the absence of exogenous stimuli. Accumulating evidence from preclinical studies and first proof-of-concept studies in patients with fibrotic disease demonstrate that targeting the aberrant epigenetic modifications may offer potential for the treatment of fibrotic diseases ${ }^{10,13-21}$.

Macroautophagy (often simply referred to as autophagy) is an evolutionarily conserved catabolic process allowing cells to degrade unnecessary or dysfunctional cellular organelles, which is an important source of nutrients during starvation or in response to cellular stress ${ }^{22}$. Moreover, components of the autophagy machinery (ATGs) are involved in secretion (secretory autophagy), including unconventional secretion of proteins ${ }^{23-26}$. Recently, LC3 has been shown to mediate the loading of protein and RNA cargoes into extracellular vesicles for secretion into the extracellular space ${ }^{27}$. Autophagy also supports other secretory pathways, such as constitutive and regulated secretion ${ }^{28}$ and may thus offers an alternative trafficking route for integral membrane proteins to the plasma membrane ${ }^{29}$.

Autophagy is initiated by formation of an isolation membrane, which elongates to sequester damaged organelles in autophagosomes. These autophagosomes subsequently fuse with lysosomes to initiate the degradation of the engulfed material ${ }^{30}$. Autophagy is tightly regulated by a complex cascade of signaling events that involve a panel of autophagy-related proteins (ATG). Key regulators that are also used as markers for autophagy include BECLIN1, the human orthologue of yeast ATG6, which promotes the formation of the isolation membrane and ATG7, which is required for elongation of the isolation membrane ${ }^{31-34}$. In parallel to the identification of key effector molecules of the autophagocytic machinery, dysregulation of autophagy has been linked to various pathophysiological conditions such as aging, cancer, inflammation and autoimmunity 35,36 . Aberrant activation of autophagy has also been implicated in the pathogenesis of fibrotic diseases. Depending on the primary effector cells in the affected organs and the kinetics of the triggering insult, pro- as well as antifibrotic effects of autophagy have been reported, suggesting a context-dependent outcome ${ }^{37-45}$. Several stimuli known to be present in SSc and other fibrotic diseases such as profibrotic and pro-inflammatory cytokines or hypoperfusion with subsequent tissue hypoxia and impaired nutrient supply are known to be capable of activating autophagy 46,47 . Recent evidence demonstrates that regulation of autophagy can also occur at an epigenetic level. The expression of several ATGs is regulated by acetylation of histone $\mathrm{H} 4$ lysine $16^{48-50}$, thus allowing to modulate the threshold for autophagy in individual cells via histone acetyltransferases. However, whether epigenetic alterations contribute to deregulation of autophagy in pathologic contexts has not yet been determined.

In the present study, we aimed to characterize the regulation of autophagy in fibrosis, to investigate, whether the epigenetic alterations in fibroblasts promote autophagy and to analyze whether targeting of autophagy in fibroblasts may prevent their aberrant activation in fibrotic diseases. We demonstrate that aberrant TGF $\beta$ signaling in fibrotic diseases perturbs the epigenetic regulation of autophagy. Re-establishment of adequate H4K16 acetylation inhibits autophagy, limits the profibrotic effects of TGF $\beta$ and ameliorates dermal and pulmonary fibrosis. Restoration of adequate epigenetic control of autophagy might thus restore tissue homeostasis and to limit fibrotic tissue remodeling.

\section{Results}

Autophagy is activated in patients with SSc and in experimental models of fibrosis. We first analyzed the activation levels of autophagy in SSc. We found that autophagy is upregulated in fibrotic skin of SSc patients with increased staining for BECLIN1 and ATG7 (Fig. 1a, b, d), two core regulators of autophagy, as compared to non-fibrotic skin. Co-staining with the fibroblast marker prolyl-4-hydroxylase- $\beta$ ( $\mathrm{P} 4 \mathrm{H} \beta)$ and the myofibroblast marker a smooth muscle actin (aSMA) demonstrated that autophagy is particularly upregulated in SSc fibroblasts (Fig. 1a, b, d). Consistently, the protein levels of p62, which correlate inversely with the activation levels of autophagy ${ }^{51}$, were downregulated in the skin of SSc patients (Fig. 1c, d). Moreover, the mRNA levels of BECLIN1 and ATG7 were increased in fibrotic SSc skin as compared to control skin (Fig. 1e). Activation of autophagy with induction of BECLIN1 and ATG7 and downregulation of p62 in fibroblasts was also observed in murine models of pulmonary (Supplementary Fig. 1a-d) or dermal fibrosis (Supplementary Fig. 2a-d). The mRNA levels of $p 62$ were not significantly altered, demonstrating that the decrease in p62 protein is not due to impaired transcription, but rather due to degradation by increased autophagic flux (Fig. 1f). Similar findings were obtained in the mouse models of bleomycin-induced fibrosis and in TBRIact-induced fibrosis (Supplementary Figs. 1e and 3a). In addition, quantification of the levels of lipidated and unconjugated LC3B confirmed an increased autophagic flux in SSc fibroblasts (Fig. 1g). To further confirm the enhanced autophagic flux in SSc, we performed co-staining for LC3B with 
a

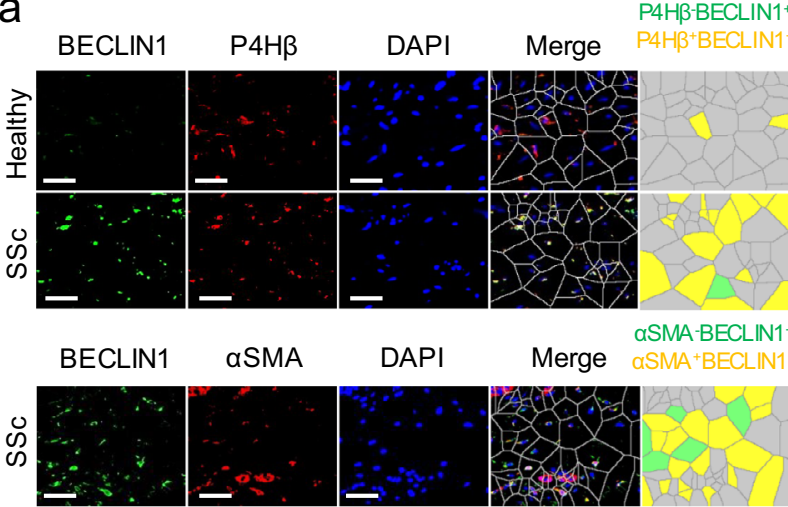

b
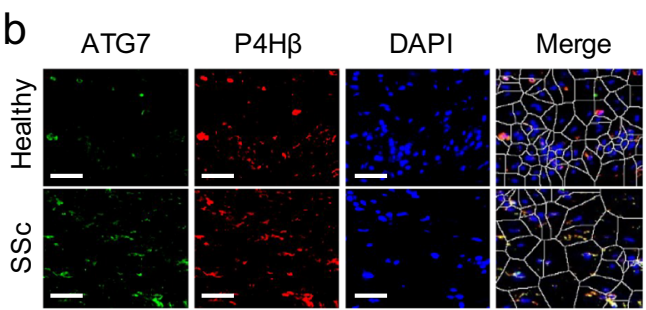

P4HB-ATG7 ${ }^{+}$
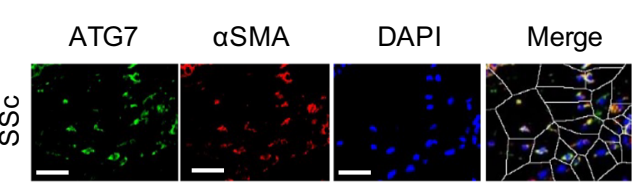

C
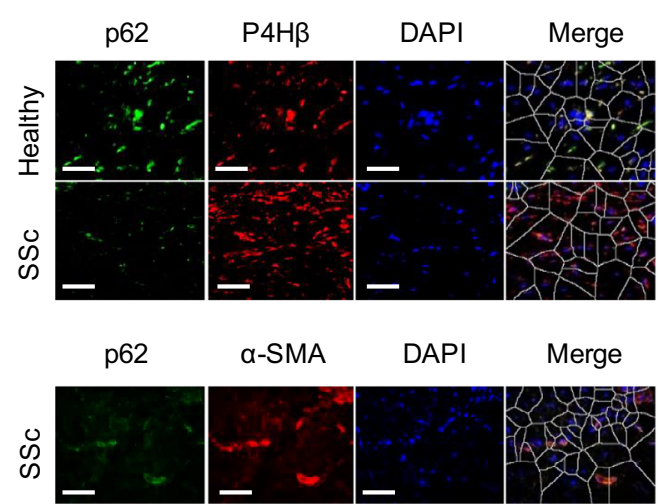

d

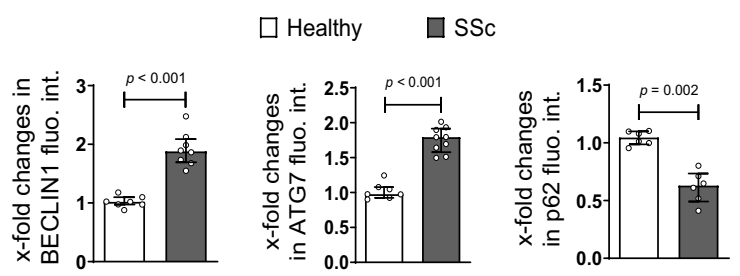

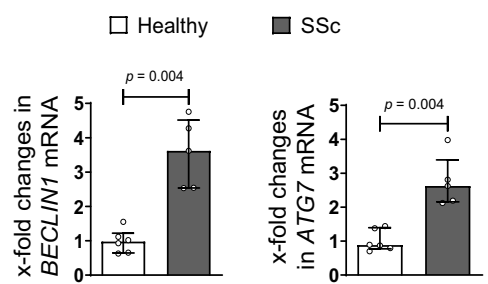

f

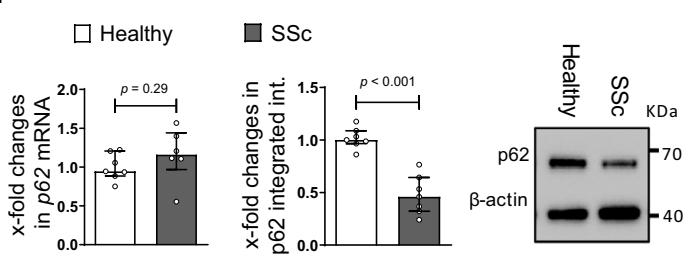

g

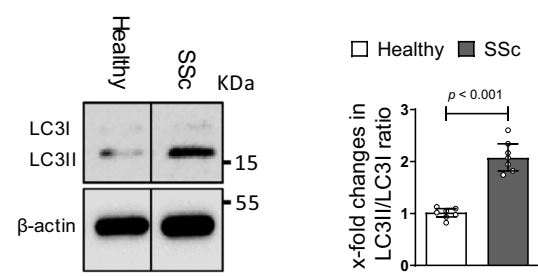

h $\quad$ LC3B
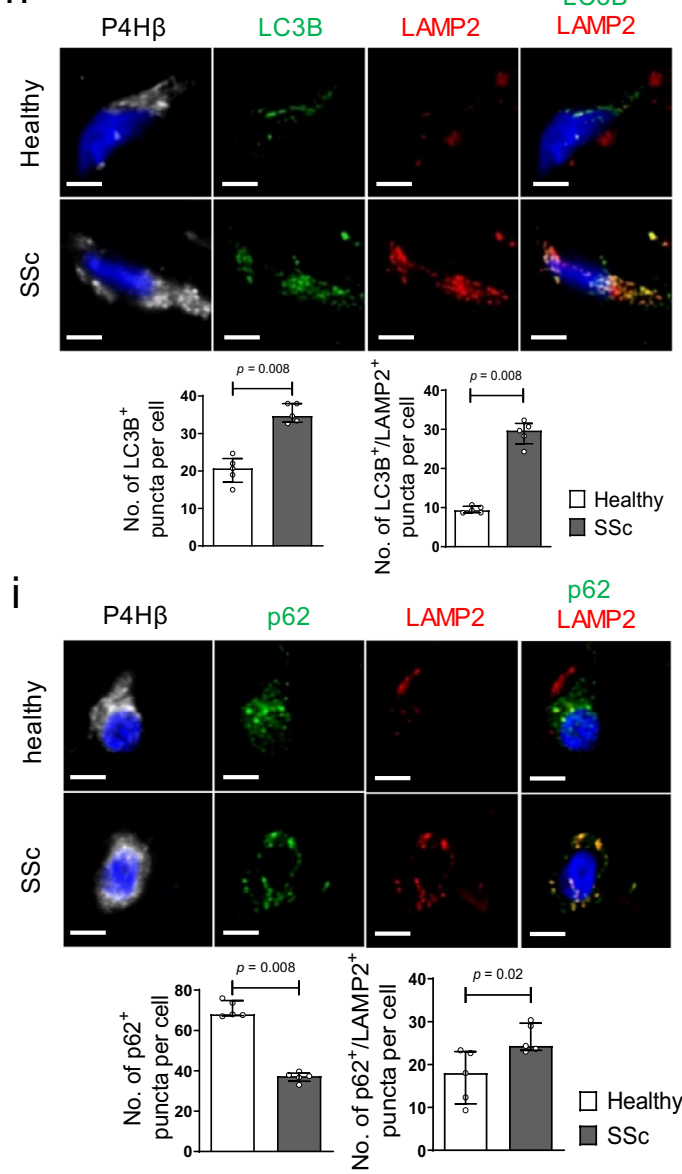

LAMP2 and p62 with LAMP2 and the fibroblast marker P4H $\beta$ in skin sections with subsequent confocal microscopy and semiautomated quantification. LC3B-positive puncta were increased in fibroblasts in SSc skin compared to healthy individuals (Fig. 1h). We observed comparable increases of LC3B puncta in murine models of SSc (Supplementary Figs. 2e, f and 3b, c). Most of the LC3B-positive puncta co-localized with the lysosomal marker LAMP2. Moreover, p62 was decreased in fibrotic tissues and most p62-positive puncta co-localized with LAMP2 (Fig. 1i).

TGF $\beta$ promotes autophagy in fibroblasts. The induction of autophagy in SSc as well as in experimental fibrosis models suggested that a common mediator of fibrosis might drive the activation of autophagy. Incubation with recombinant TGF $\beta$ 
Fig. 1 Autophagy is activated in fibrotic conditions. a-d SSc skin. a-d Representative immunofluorescence staining of BECLIN1 (a; $n=7$ healthy individuals and $n=8$ SSc patients), ATG7 ( $\mathbf{b} ; n=7$ healthy group and $n=9$ for SSc group) or p62 (c; $n=6$ patients per group) as markers of autophagy (all green) in combination with DAPI (blue) and the fibroblast marker $\mathrm{P} 4 \mathrm{H} \beta$ or the myofibroblast marker $\alpha \mathrm{SMA}$ (all red) with respective quantifications (d) and Voronoi mesh-based tessellated images. Horizontal scale bars, $50 \mu \mathrm{m}$. e mRNA levels of BECLIN1 and ATG7 (both with $n=6$ patients for healthy and $n=5$ for SSc). $\mathbf{f}$ mRNA ( $n=7$ biological replicates for healthy and $n=6$ for SSc) and protein ( $n=7$ biological replicates per group) levels of p62 in cultured human skin fibroblasts. $\mathbf{g}$ Ratio of LC3 II to LC3 I in SSc fibroblasts and controls with representative western blots and quantification ( $n=7$ biological replicates per group). h-i SSc skin. $\mathbf{h}$ Co-staining of LC3B (green) and LAMP2 (red) in combination with DAPI (blue) and the fibroblast marker $\mathrm{P} 4 \mathrm{H} \beta$ (gray) with representative confocal images and quantifications ( $n=5$ biological replicates per group). i Co-staining of p62 (green) and LAMP2 (red) in Combination with DAPI (blue) and the fibroblast marker P4H $\beta$ (gray) ( $n=5$ biological replicates per group): Representative confocal images and quantifications. Horizontal scale bars, $5 \mu \mathrm{m}$. All data are presented as median \pm IQR. $p$-values were determined by two-sided Mann-Whitney test and are indicated in the figure. See source data for more detailed information. Int.: intensity, fluo.: fluorescence., SSc: systemic sclerosis, $\mathrm{P} 4 \mathrm{H} \beta$ : prolyl 4 hydroxylase. Western blot samples in panel $\mathbf{g}$ were run on the same gel. Images were cropped at the lines only for the purpose of this figure.

increased the mRNA (Fig. 2a) and protein (Fig. 2b) levels of BECLIN1 and ATG7 and reduced the expression of p62 in cultured human fibroblasts. Stimulation of fibroblasts with TGF $\beta$ also promoted the conversion of LC3 I to LC3 II with increased ratios of LC3 II to LC3 I (Fig. 2c) and enhanced the intensity of acridine staining (Supplementary Fig. 4a). Starvation and incubation with bafilomycin A1 (BafA1) served as controls. Moreover, TGF $\beta$ reduced EGFP/RFP-LC3 reporter activity ${ }^{52}$ as additional readouts for activated autophagy (Fig. 2d). Furthermore, stimulation of fibroblasts with TGF $\beta$ promoted the formation of autophagosomes as demonstrated by electron microscopy (Fig. 2e). Immunostaining for LC3B in the presence and absence of BafA1 and TGF $\beta$ revealed that human fibroblasts exposed to TGF $\beta$ have increased levels of LC3B, which further increases in the presence of BafA1, suggesting increased autophagic flux after TGF $\beta$ stimulation compared to controls (Supplementary Fig. $4 \mathrm{~b}$ ).

The induction of autophagy by TGF $\beta$ was mediated by canonical TGF $\beta /$ SMAD3 signaling. siRNA-mediated knockdown of SMAD3 prevented the TGF $\beta$-induced upregulation of ATG7 and BECLIN1 mRNA and protein in fibroblasts (Supplementary Fig. 4c).

Moreover, activation of TGF $\beta$ signaling in vivo by overexpression of a constitutively active TGF $\beta$ receptor type I (T $\beta$ RIact) in murine skin (Fig. 2f, g) or lungs (Supplementary Fig. $4 \mathrm{~d}$, e) activated autophagy with increased mRNA and protein levels of BECLIN1 and ATG7 and downregulated p62 expression. Overexpression of T $\beta$ RIact also modulated EGFP/RFP-LC3 reporter activity in mice (Fig. 2h). In contrast, when TGF $\beta$ signaling was selectively inhibited in experimental fibrosis using the TRRI inhibitor SD-208, the induction of autophagy in bleomycin-induced fibrosis was abrogated and the levels of BECLIN1, ATG7 and p62 were comparable to those of nonfibrotic controls (Supplementary Fig. 5).

TGF $\beta$ activates autophagy by repression of MYST1. Given the accumulating evidence for epigenetic alterations in the pathogenesis of fibrotic disease, we hypothesized that TGF $\beta$ might activate autophagy by an epigenetic mechanism. To evaluate the role of different epigenetic modifications, we selectively inhibited individual epigenetic modifications and analyzed the effects on the TGF $\beta$ induced EGFP/RFP-LC3 reporter activity. Inhibition of histone deacetylases (HDACs) by trichostatin A (TSA) or by vorinostat (SAHA) abrogated TGF $\beta$-induced changes in EGFP/RFP-LC3 reporter activity, whereas inhibition of DNA methyltransferases or of H3K27 histone methyltransferases had no significant effects on TGF $\beta$-induced reporter activity (Supplementary Fig. 6a). The results indicate that histone acetylation plays a crucial role for the stimulatory effects of TGF $\beta$ on autophagy. To further investigate, how TGF $\beta$ alters histone acetylation to regulate autophagy, we first analyzed whether TGF $\beta$ induces the expression of HDACs in fibroblasts and whether the expression levels of HDACs differ between fibroblasts from SSc patients and healthy donors. Next, we examined potential effects of TGF $\beta$ on the expression of histone acetyltransferases. Given the plethora of enzymes with histone acetyltransferase activity, we first focused on the H4K16 histone acetyltransferase MYST1, which has been shown to be capable of modulating the transcription of autophagy-related genes ${ }^{50,53}$. We first confirmed that overexpression of MYST1 by adenoviral vectors induces H4K16 acetylation in cultured fibroblasts (Supplementary Fig. 6b).

Overexpression of MYST1 in fibroblasts abrogated the stimulatory effects of TGF $\beta$ on EGFP/RFP-LC3 reporter activity, whereas siRNA-mediated knockdown of MYST1 enhanced the activity (Supplementary Fig. 6c).

We next analyzed whether TGF $\beta$ regulates MYST1 by modulating its expression. TGF $\beta$ downregulated the mRNA and protein levels of MYST1 in cultured human fibroblasts (Fig. 3a and Supplementary Fig. 7a). TGF $\beta$ signaling also regulated the expression of MYST1 in vivo with decreased levels of MYST1 in murine lungs (Supplementary Fig. 7b) and skin (Supplementary Fig. 7c) upon forced expression of T $\beta$ RIact.

The repressive effects of TGF $\beta$ were dependent on canonical SMAD signaling. In silico analysis of the human MYST1 promoter revealed three SMAD binding elements (SBE). siRNA-mediated knockdown of SMAD3 (Fig. 3b) or incubation with the SMAD3 inhibitor SIS3 (Supplementary Fig. 7d, e) abrogated the repressive effects of TGF $\beta$ on MYST1. Individual analyses of the three predicted SBEs by ChIP assays (Fig. 3c) revealed that TGF $\beta$ strongly induced binding of SMAD3 to two SBE at residues 279-282 and at residues 384-387 (referred to SBE1 and SBE2, respectively) of MYST1 promoter sequence, but had only minor effects on the predicted binding site at residues 574-577 (referred to as SBE3) (Fig. 3c). Consistently, mutations of the SBE1 and the SBE2, but not at the SBE3, prevented the inhibitory effects of TGF $\beta$ in MYST1 reporter assays (Fig. 3d).

To provide evidence that this epigenetic regulation is operative in fibrotic conditions, we assessed the expression of MYST1 in skin sections of SSc patients. The mRNA and protein levels of MYST1 were decreased in SSc skin compared to non-fibrotic skin from healthy donors (Fig. 3e). Reduced MYST1 expression was also observed in bleomycin-induced dermal (Fig. 3f) and pulmonary (Supplementary Fig. 7f, g) fibrosis.

To demonstrate that downregulation of MYST1 is sufficient to activate autophagy in human fibroblasts, MYST1 expression was targeted by siRNA. Downregulation of MYST1 expression, to levels comparable to those in TGF $\beta$-stimulated human fibroblasts, activated autophagy with increased levels of ATG7 and BECLIN1 mRNA and protein, downregulation of p62 (Fig. 4a, b) and reduced EGFP/RFP-LC3 reporter activity (Fig. 4c). In contrast, overexpression of MYST1 in human fibroblasts abrogated the stimulatory effects of TGF $\beta$ on autophagy (Fig. $4 \mathrm{~d}$, e). To confirm that the antifibrotic effects of MYST1 are mediated via autophagy, we overexpressed BECLIN1 and simultaneously knocked down MYST1 in fibroblasts. Knockdown of MYST1 
a
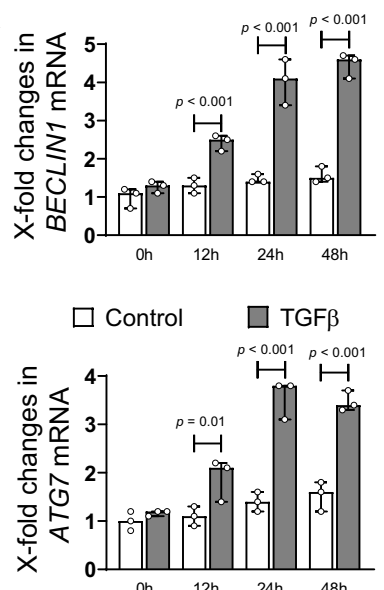

b

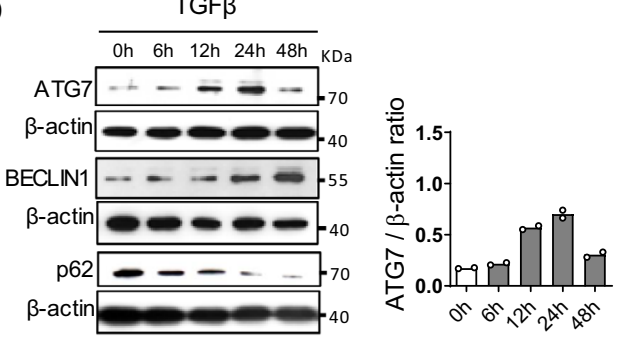

$\square$ Control $\square$ TGF $\beta$
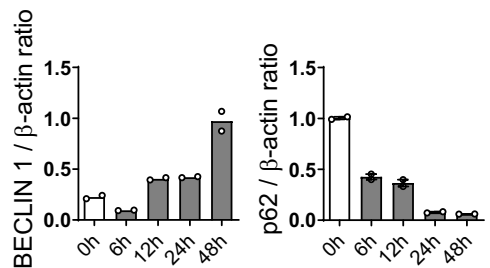

C

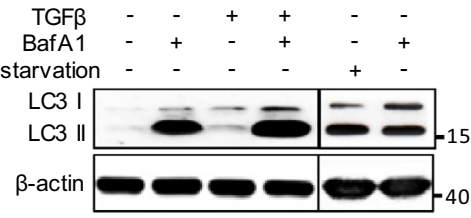

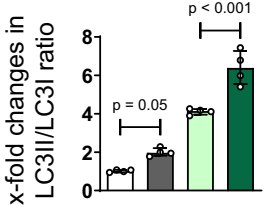

BafA1

$\square$ TGF $\beta$ $\square$ BafA1+TGF $\beta$ d

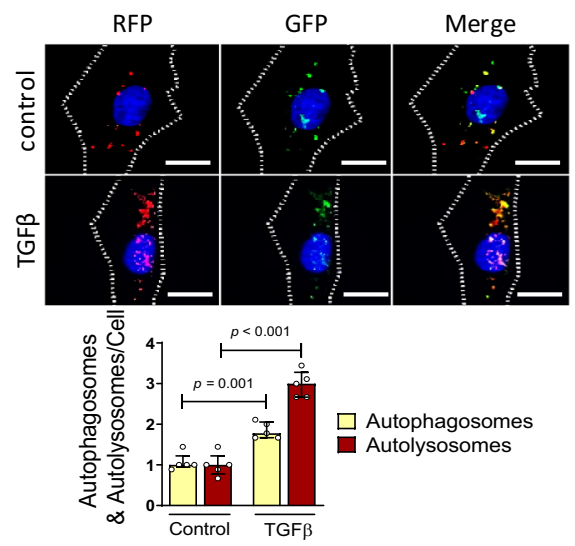

9
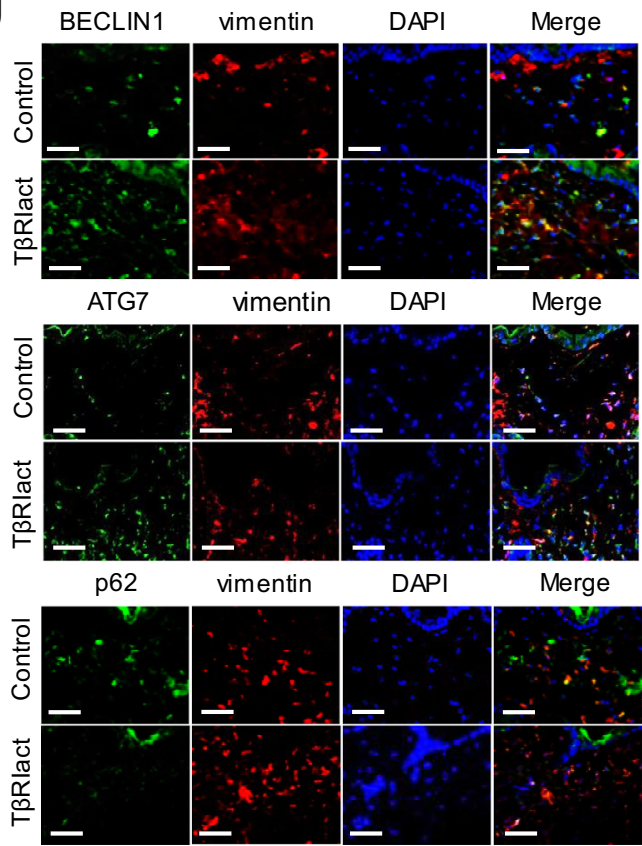

e

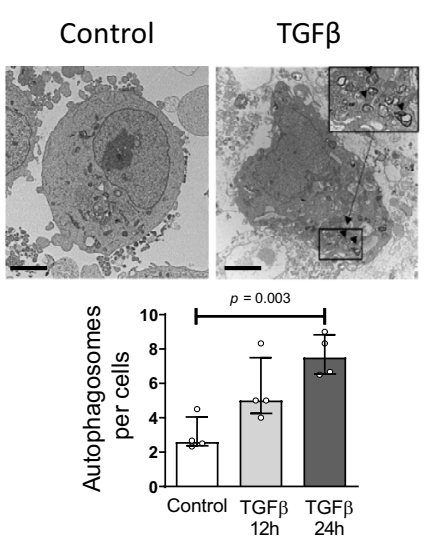

f

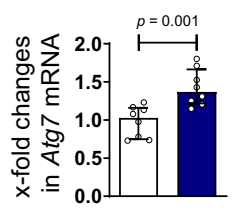

$\square$ Control $\square$ T $\beta$ Rlact

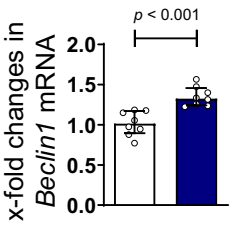

$\mathrm{h}$

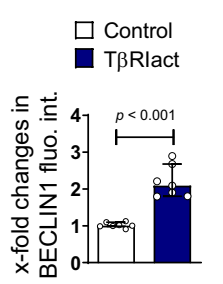

h
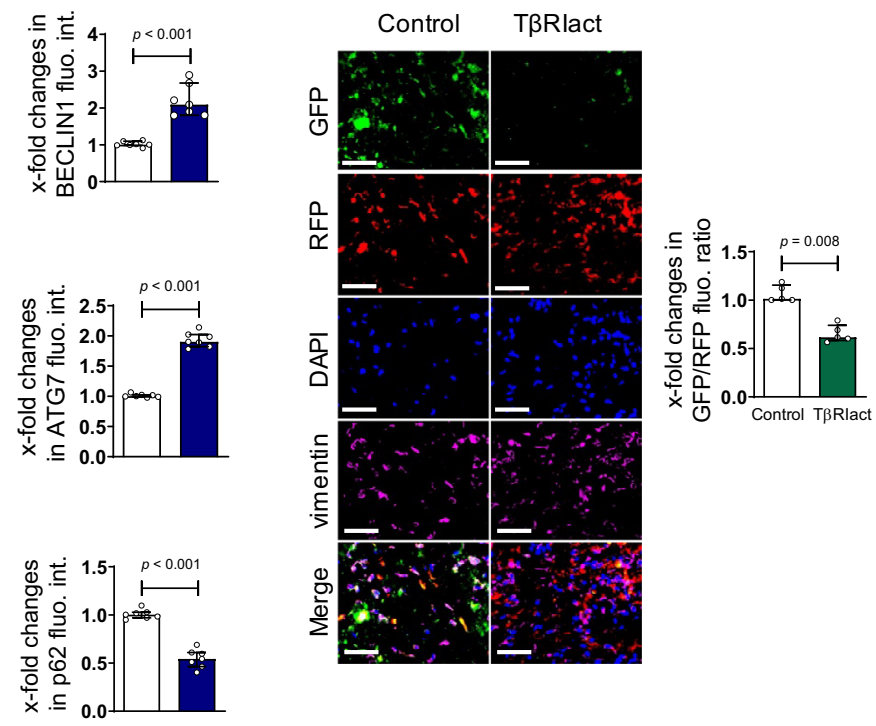

further amplified the stimulatory effects of BECLIN1 overexpression on fibroblasts with higher levels of COL1A1 mRNA and type I collagen protein (Supplementary Fig. 7h).

Activation of autophagy stimulates fibroblast activation and induces fibrosis. We next aimed to determine whether upregulation of autophagy may contribute to the activated phenotype of SSc fibroblasts and promote collagen release. Overexpression of BECLIN1 induced autophagy (Fig. 5a and Supplementary Fig. 8a) and promoted the differentiation of resting fibroblasts into myofibroblasts with increased expression of $\alpha$-smooth muscle actin (aSMA) and formation of stress fibers (Fig. 5b). Overexpression of BECLIN1 also increased the mRNA 
Fig. 2 Autophagy is activated in a TGF $\beta$-dependent manner in fibrosis. a-e Activation of autophagy in cultured fibroblasts by TGF $\beta$. a mRNA levels of BECLIN1 and ATG7 ( $n=3$ samples per group). b Protein levels of ATG7, BECLIN1, and p62. Representative western blots as well as the quantifications ( $n=$ 2 biological replicates per group over two independent experiments). c Ratio of LC3 II to LC3 I: Representative western blots and quantification. Bafilomycin and starvation served as control ( $n=4$ samples per group). $\mathbf{d}$ EGFP/RFP-LC3 reporter activity; representative images and quantification ( $n=5$ biological replicates per group). Horizontal scale bar, $5 \mu \mathrm{m}$. e Representative electron microscopy images at 12,000-fold magnification (horizontal scale bars, $2 \mu \mathrm{m} ; n$ $=4$ biological replicates) and quantification. Autophagosomes are indicated by arrows. $\mathbf{f}-\mathbf{h}$ Overexpression of T $\beta R$ lact in murine skin. $\mathbf{f}$ mRNA levels of Beclin1 and Atg7 ( $n=8$ biological replicates per group). $\mathbf{g}$ Representative immunofluorescence staining of BECLIN1, ATG7 or p62 (all green) in combination with DAPI (blue) and vimentin (red; $n=7$ biological replicates per group for all experiments). $\mathbf{h}$ Representative images and quantification of EGFP/RFP-LC3 reporter activity in the skin ( $n=5$ biological replicates for both groups). Horizontal scale bars, $50 \mu \mathrm{m}$. All data are presented as median $\pm I Q R$. $p$-values were determined by ANOVA one-way with Sidak's multiple comparisons test (a) or with Tukey's multiple comparison post hoc test (c-e) and by two-sided Mann-Whitney test (f-h). $p$-values are indicated in the figure. See source data for more detailed information. Western blot samples in panel $\mathbf{c}$ cropped at the black horizontal line were not run on the same gel. Images were cropped at the line only for the purpose of this figure. int.: intensity, fluo.: fluorescence, T $\beta$ Rlact: constitutively active TGF $\beta$ receptor type I. BafA1: bafilomycin A1, fluo.: fluorescence.

levels of COL1A1 and stimulated the release of type I collagen protein and total collagen protein into the supernatant (Fig. 5c). In contrast, co-incubation of TGF $\beta$ stimulated human dermal fibroblasts with chloroquine (CQ), a pharmacological inhibitor of autophagy, decreased the mRNA levels of COL1A1 and reduced the secretion of collagen protein (Supplementary Fig. 8b).

Overexpression of BECLIN1 also activated autophagy in vivo and induced fibrosis in the skin and lungs of mice in the absence of additional profibrotic stimuli. Overexpression of BECLIN1 in the lungs of mice activated autophagy (Fig. 5d) and induced myofibroblast differentiation with accumulation of collagen and pulmonary fibrosis (Fig. 5e). Overexpression of BECLIN1 was also sufficient to induce autophagy, myofibroblast differentiation and fibrosis in the skin of mice (Supplementary Fig. 8c, d). To further validate that autophagy regulates the deposition of extracellular matrix, we quantified extracellular matrix (ECM) deposition in fibroblasts overexpressing BECLIN1 and in fibroblasts treated with the autophagy inhibitor 3-methyladenine (3-MA) (Supplementary Fig. 9a, b). Overexpression of BECLIN1 promoted deposition of collagen I, collagen III and fibronectin, with effects comparable to that of TGF $\beta$ (Supplementary Fig. 9a).

Recent evidence demonstrated that the autophagic machinery is required for secretion of TGF $\beta 1$ and that inhibition of autophagy blocks the release of $\mathrm{TGF} \beta 1^{26}$. We therefore investigated whether MYST1 may ameliorate fibroblast activation and tissue fibrosis by inhibition of TGF $\beta$ signaling. Overexpression of BECLIN1 induced the mRNA levels of the prototypical TGF $\beta / S M A D$ target genes $P A I-1$ and CTGF in human dermal fibroblasts (Supplementary Fig. 10a). In contrast, inhibition of autophagy by overexpression of MYST1 reduced the stimulatory effects of TGF $\beta$ on PAI-1 and CTGF mRNA (Supplementary Fig. 10b). Consistently, overexpression of BECLIN1 increased the levels of active TGF $\beta$ released from dermal fibroblasts, whereas overexpression of MYST1 decreased them (Supplementary Fig. 10c). Overexpression of BECLIN1 also promoted TGF $\beta$ signaling in murine skin with increased levels of pSMAD3 and of active TGF $\beta$ in western blot and in MLC assays (Supplementary Fig. 10d, e). Overexpression of MYST1 ameliorated TGF $\beta$ signaling with reduced levels of pSMAD3 and of active TGF $\beta$ in bleomycin-challenged mice (Supplementary Fig. 10f, g).

Inhibition of autophagy by fibroblast-specific knockout of Atg7 reduces fibroblast activation and ameliorates experimental fibrosis. We next investigated whether inactivation of autophagy can inhibit TGF $\beta$-induced fibroblast-to-myofibroblast differentiation. Indeed, fibroblasts lacking Atg7 were less sensitive to the profibrotic effects of TGF $\beta$ with decreased expression of aSMA and stress fibers (Fig. 6a) and reduced release of collagen protein as compared to control fibroblasts (Fig. 6b). Moreover, we analyzed the levels of type I collagen released by human fibroblasts with stable knockout of ATG7 using SILAC-based quantitative proteomics (Supplementary Fig. 11a). Inhibition of autophagy by knockout of ATG7 ameliorates the release of both type I collagen chains in the supernatant. The differences in type I collagen release were confirmed by western blot (Supplementary Fig. 11b). We also observed impaired TGF $\beta$ release and activation upon ATG7 knockout by SILAC-based proteomics (Supplementary Fig. 11a) and in cells expressing FLAG-tagged TGF $\beta$ (Supplementary Fig. 11c).

In addition, inhibition of autophagy by 3-MA inhibited TGF $\beta$ induced myofibroblast differentiation as well as TGF $\beta$-induced deposition of type I and type III collagen and of fibronectin (Supplementary Fig. 9b and 12a).

We next analyzed whether inactivation of autophagy can ameliorate experimental fibrosis. We first demonstrated that treatment of mice with the autophagy inhibitor 3-MA can ameliorate bleomycin-induced skin fibrosis (Supplementary Fig. 12b).

We next generated Atg $7 \mathrm{fl} / \mathrm{fl} \times$ Colla2;CreER mice and $A \operatorname{tg} 7 \mathrm{fl} / \mathrm{fl} \times$ Col6;Cre for selective inactivation of Atg7 in fibroblasts 8,54 . Inactivation of autophagy in fibroblasts ameliorated TGF $\beta$ dependent experimental pulmonary and dermal fibrosis. Fibroblastspecific knockout of Atg7 reduced the TßRIact-induced increase in Ashcroft scores and in fibrotic area as well as myofibroblast counts and hydroxyproline content compared to mice with normal expression of Atg7 in experimental pulmonary fibrosis (Supplementary Fig. 13). Inactivation of Atg7 also prevented TRRIact-induced skin fibrosis, demonstrating that the activation of autophagy directly contributes to the profibrotic effects of TGF $\beta$ (Fig. $6 \mathrm{c}$ ). In addition to T $\beta$ RIact-induced fibrosis, mice with fibroblast-specific knockout of Atg7 were also less sensitive to the profibrotic effects of intratracheal or subcutaneous application of bleomycin with less fibrotic changes on histology, reduced myofibroblast counts and lower hydroxyproline content (Fig. 6d, e).

We next investigated whether inhibition of autophagy can deactivate myofibroblasts and induce regression of tissue fibrosis. Knockdown of $A T G 7$ or BECLIN-1 in human fibroblasts reduced the expression of aSMA and the number of stress fibers in myofibroblasts, indicating re-differentiation of myofibroblasts into resting fibroblasts upon inhibition of autophagy (Fig. 7a, b,). Similar results were obtained with the autophagy inhibitors CQ and 3-MA (Supplementary Fig. 14a, b).

Moreover, we evaluated the effects of autophagy inhibition in a model of established bleomycin-induced skin fibrosis (Fig. 7c-e). In this model, fibrosis was induced by injections of bleomycin for four weeks. After these four weeks, we inhibited autophagy by siRNA-mediated knockdown of Atg7, while continuing to challenge the mice with bleomycin for additional three weeks. 
a

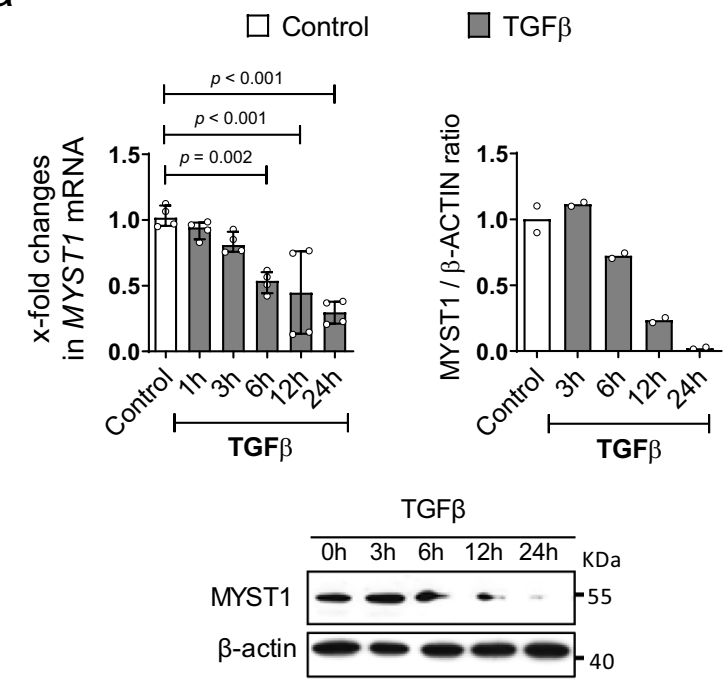

C

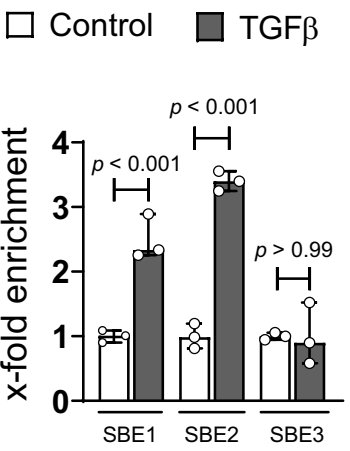

e

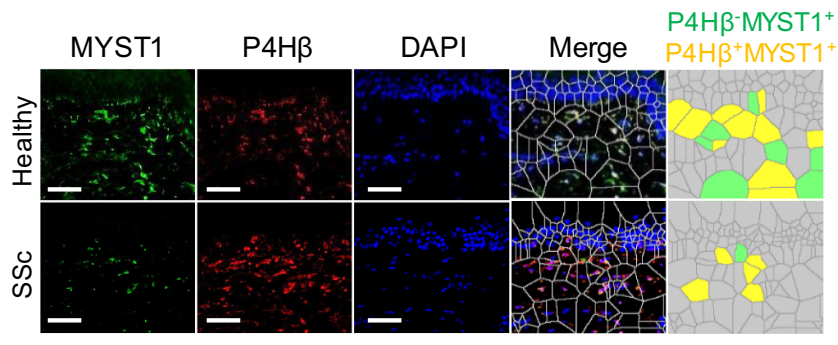

f

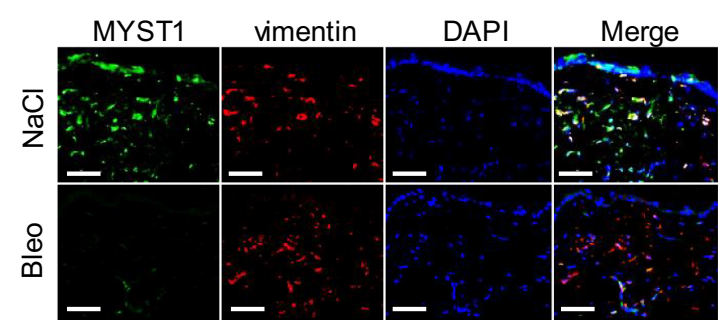

b
$\square$ Control siRNA
$\square$ Control siRNA+TGF $\beta$
$\square$ SMAD3 SIRNA
- SMAD3 SiRNA+TGF $\beta$
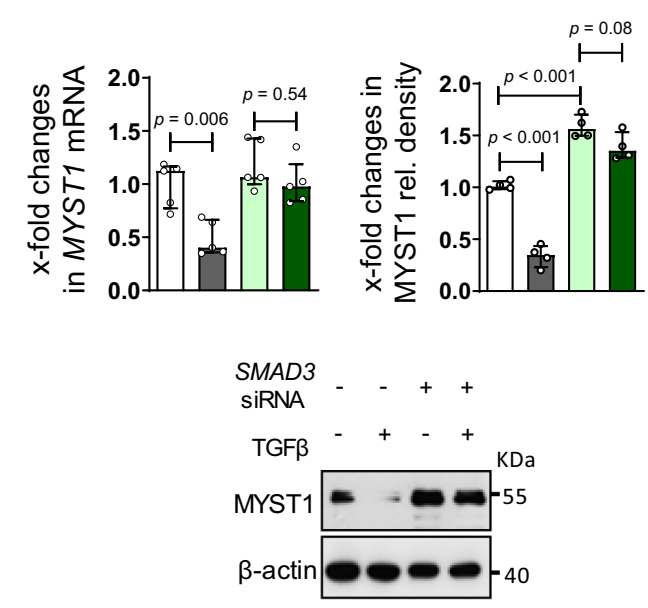

d
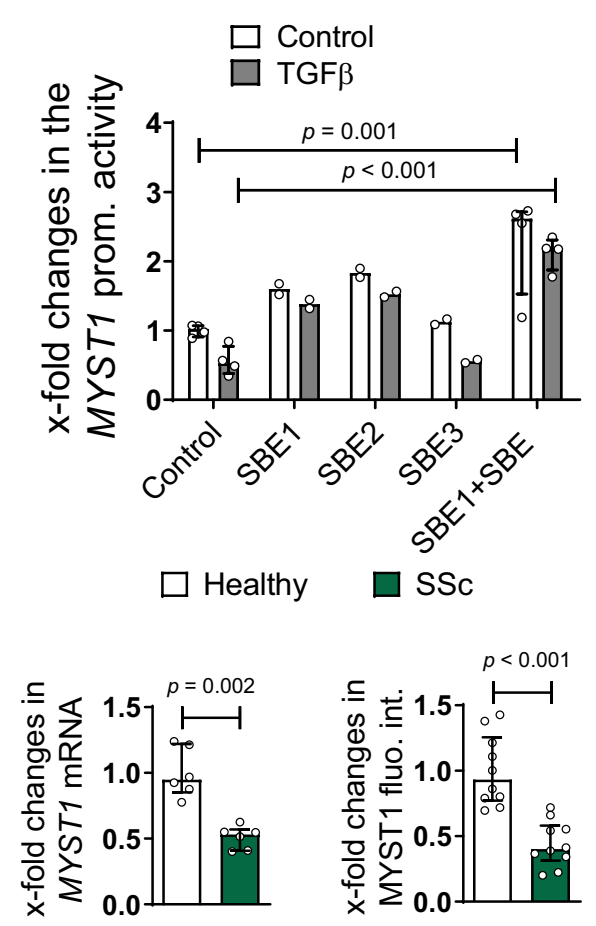

$\square \mathrm{NaCl}$

Bleo
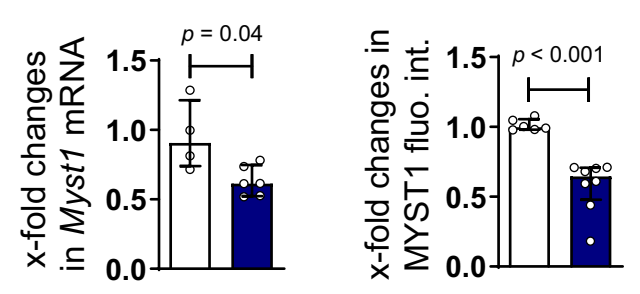

Challenge of mice with bleomycin for four weeks induced robust fibrosis, with further progression of skin fibrosis in mice challenged for additional three weeks (Fig. 7c). siRNA-mediated knockdown of $A t g 7$ effectively prevented progression of fibrosis with reduced dermal thickness, myofibroblast counts and hydroxyproline content (Fig. 7d). Of note, inactivation of autophagy also decreased dermal thickness, myofibroblast counts and hydroxyproline content to below pretreatment levels, indicating regression of bleomycin-induced skin fibrosis.

Overexpression of MYST1 ameliorates experimental fibrosis. Based on the potent inhibitory effects of MYST1 on autophagy 
Fig. 3 TGF $\beta$ downregulates MYST1 in fibroblasts. a mRNA ( $n=4$ biological replicates per group) and protein ( $n=2$ biological replicates per group over two independent experiments) levels of MYST1 in cultured fibroblasts stimulated with TGF $\beta$. $\mathbf{b}$ Effects of siRNA-mediated knockdown of SMAD3 on mRNA ( $n=5$ biological replicates per group) and protein ( $n=4$ biological replicates per group) levels of MYST1 in TGF $\beta$-stimulated fibroblasts. c Chromatin immunoprecipitation assay for binding of SMAD3 at three putative SMAD binding Elements (SBE) in the MYST1 promoter ( $n=3$ biological replicates). d Promoter assays using MYST1 promoter constructs with selective mutations at three putative SBEs, $(n=2$ biological replicates for SBE1, SBE2, and SBE3 groups and $n=4$ for the other groups). e Expression of MYST1 in SSc patients and healthy individuals analyzed by qRT-PCR ( $n=6$ patients per group) and immunofluorescence staining with Voronoi mesh-based tessellated images ( $n=10$ patients per group). $\mathbf{f}$ Expression of MYST1 in fibrotic skin of mice challenged with bleomycin or non-fibrotic control mice as analyzed by qRT-PCR ( $n=4$ biological replicates for control group and $n=6$ for bleomycin group) and immunofluorescence staining ( $n=6$ biological replicates for control group and $n=8$ for bleomycin group). All horizontal scale bars, $50 \mu$ m. All data are presented as median \pm IQR. $p$-values were determined by ANOVA one-way with Sidak's multiple comparisons test (a, $\mathbf{c}$ ) or with Tukey's multiple comparison post hoc test $(\mathbf{b}, \mathbf{d})$ and by two-sided Mann-Whitney test $(\mathbf{e}, \mathbf{f})$. p-values are indicated in the figure. See source data for more detailed information. rel.: relative, P4H $\beta$ : prolyl 4-hydroxylase, SSc: systemic sclerosis, Bleo: bleomycin, fluo.: fluorescence, int,: intensity, prom.: promoter, IP: immunoprecipitation.

and its strongly decreased expression in SSc, we hypothesized that forced expression of MYST1 may exert antifibrotic effects by preventing TGF $\beta$-induced activation of autophagy. To test this hypothesis, we first assessed the effects of MYST1 overexpression on the TGF $\beta$-dependent activation of human dermal fibroblasts. Overexpression of MYST1 reduced the TGF $\beta$-induced fibroblastto-myofibroblast transition with decreased expression of aSMA and impaired formation of stress fibers (Fig. 8a). Consistently, the levels of COL1A1 mRNA and collagen protein were reduced in fibroblasts overexpressing MYST1 compared to control cells (Fig. 8b).

Overexpression of MYST1 also ablated the activation of autophagy in T $\beta$ RIact-induced skin fibrosis. T $\beta$ RIact-induced changes in expression levels of ATG7, BECLIN1 and p62 were prevented in MYST1-overexpressing mice and the levels of these markers of autophagy were comparable to those of non-fibrotic control mice (Supplementary Figs. 15a, b and 16a). The inhibitory effects of MYST1 on autophagy translated into potent antifibrotic effects. Adenoviral overexpression of MYST1 ameliorated $T \beta R$ Iact-induced skin fibrosis with reduced dermal thickening, lower myofibroblast counts and decreased hydroxyproline content as compared to T $\beta$ RIact-challenged mice infected with control adenoviruses encoding LacZ (Fig. 8c). Overexpression of MYST1 also prevented activation of autophagy in the lungs and skin of bleomycin-challenged mice (Supplementary Figs. 16b, c, 17a, b, and $18 \mathrm{a}, \mathrm{b})$. Bleomycin-induced fibrosis was significantly less severe in both organs of mice infected with adenoviruses encoding Myst1 as compared to controls (Fig. 8d, e).

To exclude that the inhibitory effects of MYST1 on fibroblast activation and tissue fibrosis are mediated by apoptosis, we first measured the activity of Caspase 3 and 7 in fibroblasts with siRNA-mediated knockdown of MYST1 or with adenoviral overexpression of MYST1. Neither knockdown nor overexpression of MYST1 altered Caspase activity in human dermal fibroblasts as compared to respective control cells (Supplementary Fig. 19a, b). We also assessed whether overexpression of MYST1 alters the levels of apoptosis in fibrotic murine tissues. The number of apoptotic cells, in particular of fibroblasts, was not altered in bleomycin- or T $\beta$ RIact-challenged mice as analyzed by TUNEL staining (Supplementary Fig. 19c-e).

\section{Discussion}

In the present study, we demonstrate that autophagy is strongly activated in fibroblasts from SSc skin and also in experimental dermal and pulmonary fibrosis as compared to respective nonfibrotic control tissue with increased expression levels of ATG7 and BECLIN1, decreased expression of p62 and enhanced activity in in vivo autophagy reporter studies. The aberrant activation of autophagy had profound stimulatory effects on fibroblasts. Activation of autophagy by forced expression of BECLIN1 promoted fibroblast-to-myofibroblast transition, induced activation of latent TGF $\beta$ and stimulated the collagen release in vitro and induced dermal and pulmonary fibrosis in vivo. Moreover, inactivation of autophagy by fibroblast-specific knockout of Atg7 prevented myofibroblast differentiation and ameliorated pulmonary and dermal fibrosis, demonstrating that activation of autophagy is both, sufficient and required, for fibroblast activation in fibrosis.

Recent studies demonstrate that autophagy is activated in several fibrotic diseases such as liver fibrosis ${ }^{37,55,56}$, renal interstitial fibrosis ${ }^{39}$, cardiac fibrosis ${ }^{42}$ and fibrosis of the peritoneum $^{45}$. However, other reports point to a downregulation in idiopathic pulmonary fibrosis (IPF) at tissue level and murine models of pulmonary fibrosis ${ }^{57-64}$ In contrast to those findings, autophagy was reported to be activated in cultured human fibroblasts derived from patients with $\mathrm{IPF}^{37}$. Moreover, autophagy was reported to be activated in a murine model of amiodarone-induced pulmonary fibrosis ${ }^{65}$. Together these findings suggest a cell- and context-specific regulation of autophagy.

We present several lines of evidence that impaired epigenetic control is a central mechanism for the aberrant activation of autophagy in fibrotic tissue remodeling. Screening of different epigenetic modifiers identified the H4K16 histone acetyltransferase MYST1 as the central epigenetic regulator of TGF $\beta$ induced autophagy, whereas selective inhibition of several other epigenetic modifications relevant to the pathogenesis of fibrotic diseases such DNA methyltransferases or of H3K27 histone methyltransferases ${ }^{10,14,16,66-68}$ had no effect. Our study is the first to link deregulation of MYST1 to the pathogenesis of fibrotic diseases. This finding supports the concept that epigenetic alterations play a critical role in the persistent activation of fibroblasts in fibrotic diseases.

MYST1 regulates autophagy by $\mathrm{H} 4 \mathrm{~K} 16$ acetylation at the promoters of autophagy-related genes such as p62/SQSTM ${ }^{49}$, which has previously been demonstrated to be associated with reduced transcription of target genes ${ }^{48}$. Under physiologic conditions, appropriate expression of MYST1 prevents uncontrolled activation of autophagy by inhibiting the expression of core components of autophagy such as ATG7 and BECLIN1. In patients with SSc as well as in experimental fibrosis, however, the expression of MYST1 is strongly downregulated, thus impairing the epigenetic control of autophagy. We demonstrate that the downregulation of MYST1 alone is sufficient to stimulate the expression of ATG7 and BECLIN1 and to promote activation of autophagy in cultured fibroblasts and in experimental fibrosis. Our findings are in agreement with a study by Hale et al..$^{50}$, which identified MYST1 as a modulator of autophagy in an siRNA-based screening approach.

In contrast, overexpression of MYST1 prevented the aberrant activation of autophagy, inhibited TGF $\beta$-induced fibroblast activation and ameliorated experimental dermal and pulmonary fibrosis in complementary murine models. Impaired control of 
a
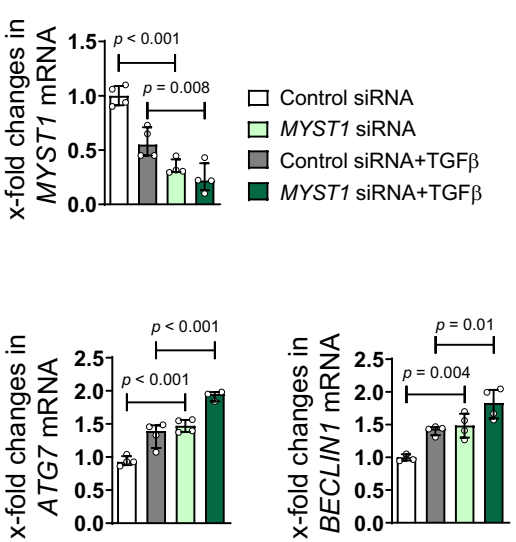

b

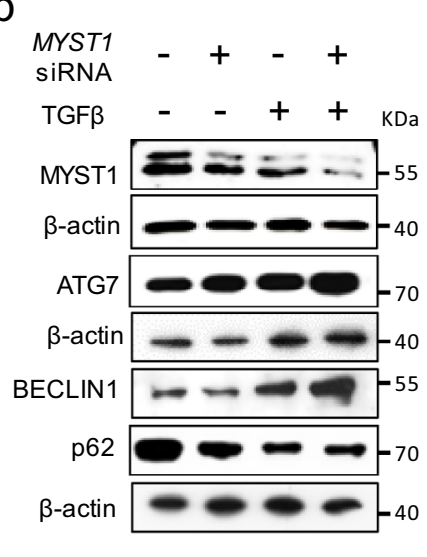

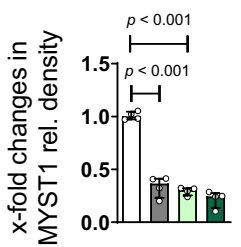

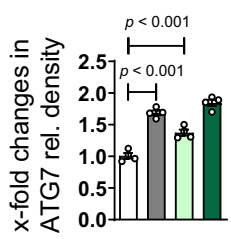

$\square$ Control siRNA

$\square$ Control siRNA +TGF $\beta$

\section{$\square$ MYST1 SiRNA}

$\square$ MYST1 SIRNA+TGF $\beta$
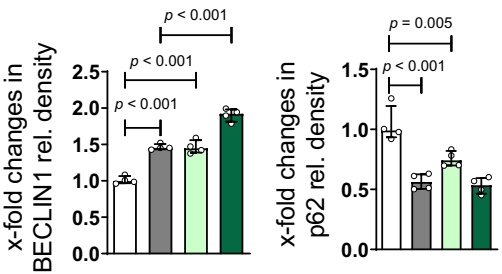

C

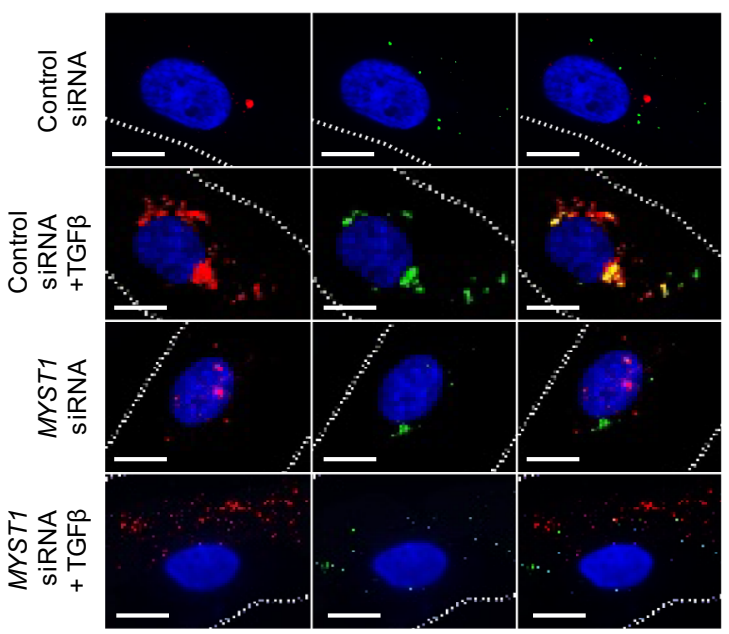

$\square$ Autophagosomes $\square$ Autolysosomes

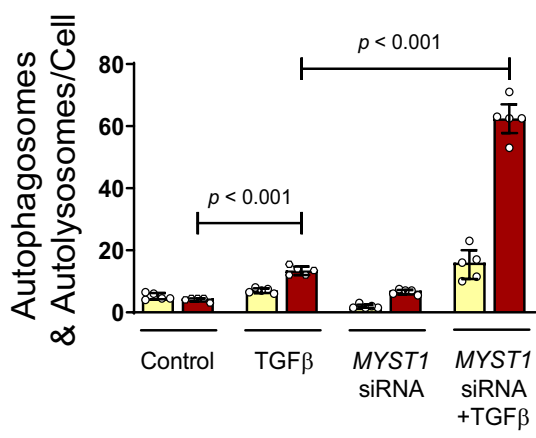

d

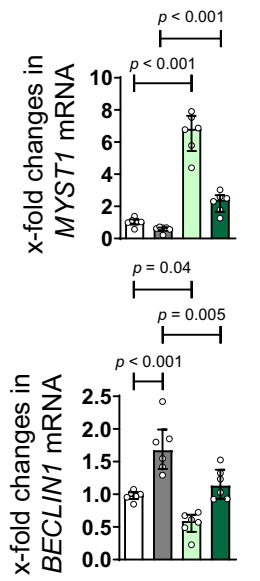

e

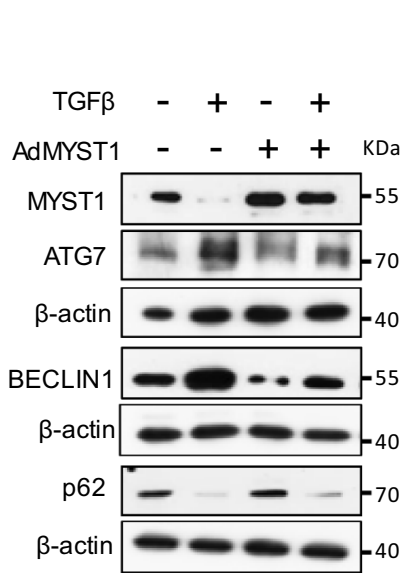

$\square$ AdLacZ

$\square$ AdLacZ + TGF $\beta$
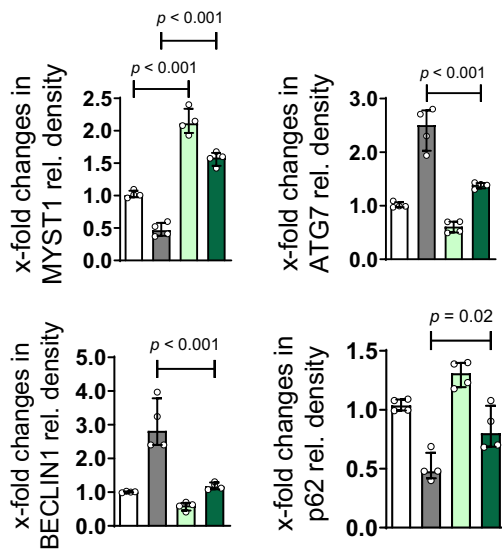

$\square$ AdMYST1+TGF $\beta$ $\square$ AdMYST1

Fig. 4 Downregulation of MYST1 mediates the stimulatory effects of TGF $\boldsymbol{\beta}$ on autophagy. $\mathbf{a}$, $\mathbf{b}$ Effects of siRNA-mediated knockdown of MYST1 on autophagy in healthy human dermal fibroblasts as assessed by quantification of the mRNA levels of ATG7 ( $n=4$ biological replicates per group and $n=3$ for MYST1 siRNA + TGF $\beta$ ), BECLIN1 ( $n=4$ biological replicates per group) and respective protein quantifications ( $n=4$ biological replicates per group). Confirmation of reduced mRNA ( $n=4$ biological replicates per group) (a) and protein ( $\mathbf{b} ; n=4$ biological replicates per group) levels of MYST1 by qRT-PCR and western blot. $\mathbf{c}$ EGFP/ RFP-LC3 reporter activity upon knockdown of MYST1; representative images and quantification ( $n=5$ biological replicates per group). Horizontal scale bar, $5 \mu$ m. $\mathbf{d}$, e mRNA expression of ATG7, BECLIN1, and MYST1 ( $n=6$ biological replicates per group) (d) and respective protein quantification (e) ( $n=4$ biological replicates per group) levels after adenoviral overexpression of MYST1 in healthy human dermal fibroblasts (e). All data are presented as mean $\pm I Q R$. $p$-values were determined by ANOVA one-way with Tukey's multiple comparison post hoc test. See source data for more detailed information. rel.: relative, Ad: adenovirus. The results in panel c are part of a large set of experiments and share controls with Supplementary Fig. 8a. Data are presented separately just for the purpose of this figure. 
a

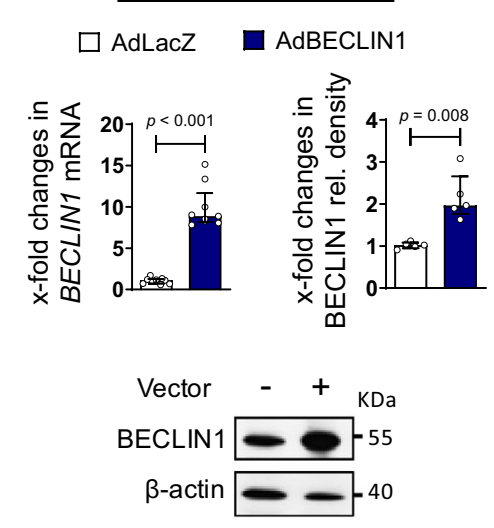

b

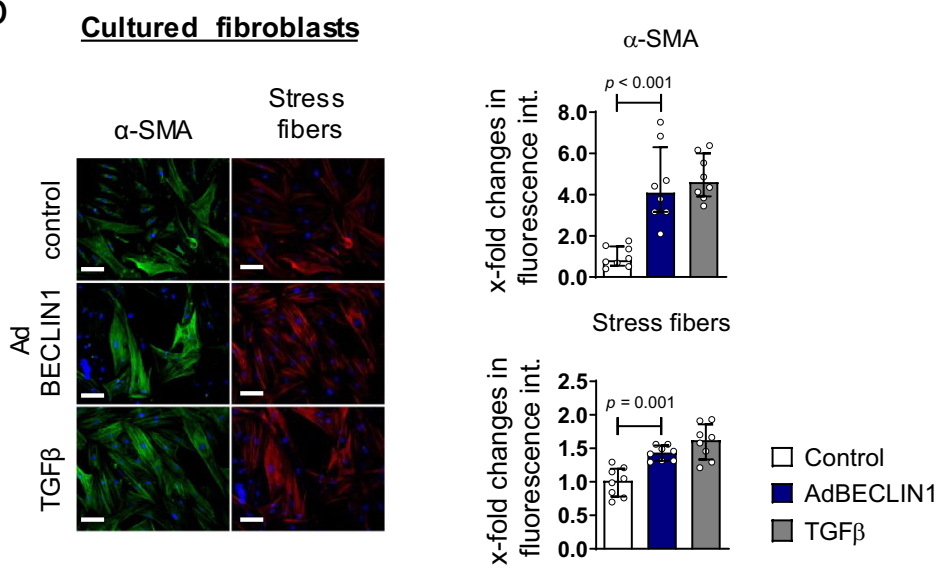

C

\section{Cultured fibroblasts}

$\square$ AdLacZ

AdBECLIN1 $\square$ TGF $\beta$
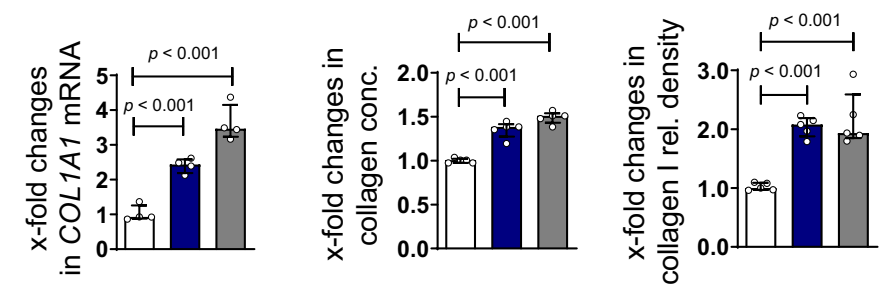

d
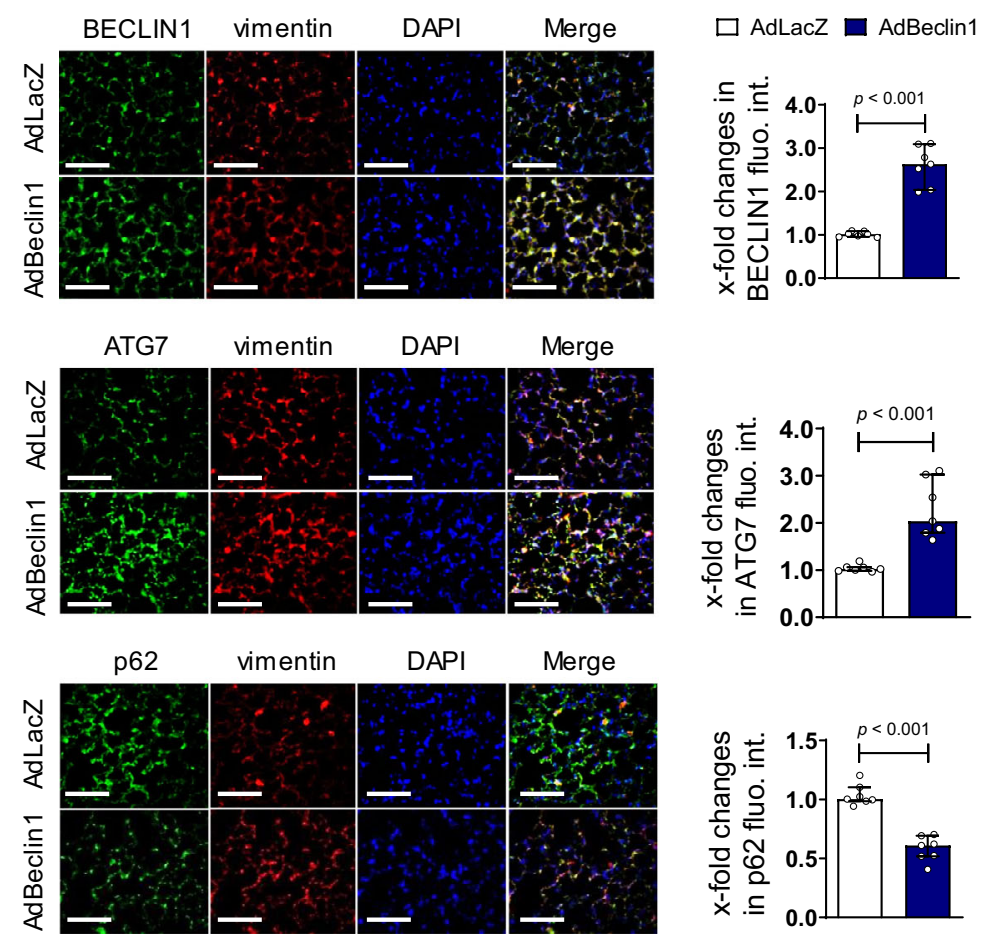

$\times$

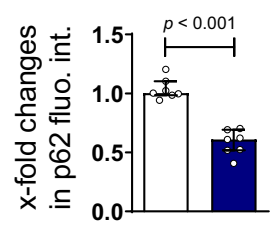

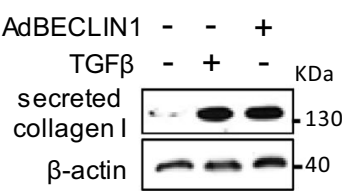

e
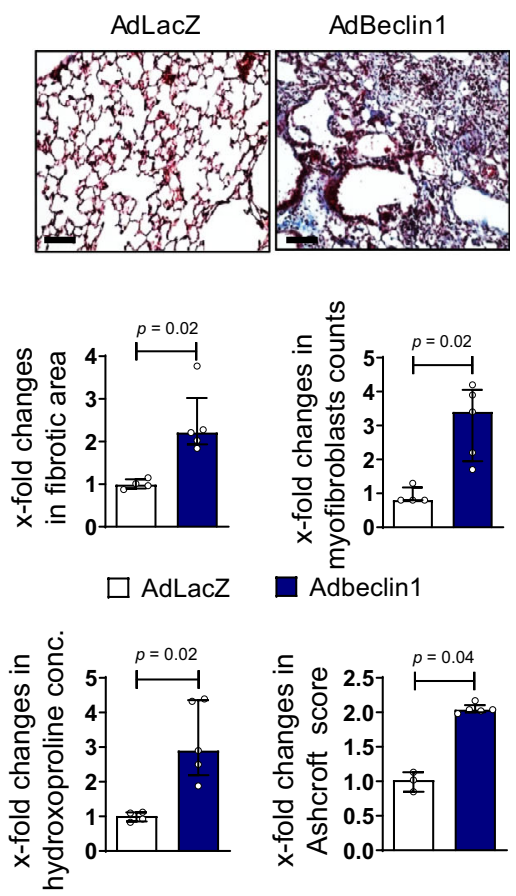

autophagy with downregulation of MYST1 may thus play a central role in the aberrant fibroblast activation and might be a potential target for therapeutic intervention in fibrotic diseases.

We demonstrate in multiple experiments that canonical TGF $\beta$ signaling is a central regulator of MYST1 expression and thus an important upstream regulator of autophagy in SSc: (I) Incubation with recombinant TGF $\beta$ decreased the mRNA and protein levels of MYST1 in cultured fibroblasts and induced autophagy. (II) Overexpression of TRRIact downregulated the expression of MYST1 in murine skin and lungs. (III) The inhibitory effects of TGF $\beta$ were mediated by canonical TGF $\beta / S M A D$ signaling and knockdown of SMAD3 abrogated the downregulation of MYST1. (IV) Treatment with SD-208, a selective inhibitor of TGF $\beta$ receptor type $1^{69}$, prevented the downregulation of MYST1 and 
Fig. 5 Autophagy induces fibroblast-to-myofibroblast transition, collagen release, and tissue fibrosis. a-c Cultured human dermal fibroblasts overexpressing BECLIN1. a Expression of BECLIN1 mRNA ( $n=9$ biological replicates per group) and protein $(n=5$ biological replicates per group) in fibroblasts infected with adenoviral vectors encoding BECLIN1 or LacZ. $\mathbf{b}$ Effects of the adenoviral overexpression of BECLIN1 on the expression of $\alpha$ SMA and the formation of stress fibers ( $n=8$ biological per group) with representative images and quantification of fluorescence intensity. Fibroblasts stimulated with recombinant TGF $\beta$ served as positive control. Horizontal scale bar, $100 \mu \mathrm{m}$. c Changes in the mRNA levels of COL1A1 as analyzed by qRTPCR ( $n=4$ biological replicates per group), on type I collagen protein as analyzed by western blot ( $n=5$ biological replicates per group) and on total collagen analyzed by hydroxyproline assays in fibroblasts upon adenoviral overexpression of BECLIN1 ( $n=5$ biological replicates per group). $\mathbf{d}$, e Adenoviral overexpression of BECLIN1 in murine lungs. d Representative immunofluorescence staining of the markers of autophagy BECLIN1, ATG7, or p62 (all green) in combination with DAPI (blue) and vimentin (red) ( $n=7$ biological replicates per group). e Trichrome stainings, fibrotic area counts, myofibroblast counts, hydroxyproline content ( $n=4$ biological replicates for control group and $n=5$ for AdBeclin1 group) and Ashcroft scores ( $n=3$ biological replicates for control group and $n=5$ for AdBeclin1 group). Horizontal scale bars, $100 \mu \mathrm{m}$. All data are presented as median \pm IQR. $p$-values were determined by two-sided Mann-Whitney test $(\mathbf{a}, \mathbf{d}, \mathbf{e})$ or ANOVA one-way with Tukey's multiple comparison post hoc test $(\mathbf{b}, \mathbf{c})$ and are indicated in the figure. See source data for more detailed information. rel.: relative, Ad: adenovirus, fluo.: fluorescence, int.: intensity, conc.: concentration.

the subsequent activation of autophagy in experimental fibrosis. Although factors such as reduced supply of nutrients and hypoxia $^{70}$ due to capillary rarefication likely contribute to the enhanced activation of autophagy in fibrotic diseases ${ }^{71}$, these findings characterize TGF $\beta$ as a central upstream regulator of autophagy in the context of fibrosis. Given the potent profibrotic effects of enhanced autophagy in fibroblasts, our study unravels the activation of autophagy as a mechanism by which TGF $\beta$ orchestrates fibroblast activation and tissue fibrosis.

Our study demonstrates that activation of autophagy promotes fibroblast-to-myofibroblast transition and that selective inactivation of autophagy ameliorates T $\beta$ RIact- and bleomycin-induced dermal and pulmonary fibrosis. However, general targeting of autophagy for the treatment of fibrosis by systemic application of inhibitors might be associated with adverse events. The major physiological role of autophagy is to provide nutrients under conditions of cellular stress and prevent cells from undergoing apoptosis. The homeostatic effects of autophagy may be of particular relevance in SSc. Apoptosis of microvascular endothelial cells with consecutive capillary rarefication, reduced perfusion and tissue hypoxia are key features of SSc. Under those conditions, direct inhibition of autophagy might further increase apoptosis of microvascular endothelial cells, aggravate microvascular disease and induce vascular complications. In contrast to approaches that interfere with autophagy directly, modulation of H4K16 acetylation via MYST1 would rather modulate the threshold for autophagy. This approach might allow maintaining the levels of autophagy that are required for homeostatic functions. Although forced re-expression of MYST1 in skin and lung was not accompanied by adverse events in our models, further studies in murine models with more extensive vascular involvement and in other organs are required to exclude vascular complications and to confirm the tolerability of targeting autophagy via MYST1.

In summary, we demonstrate that the epigenetic control of autophagy is disturbed by a TGF $\beta$-dependent downregulation of MYST1 in SSc as a typical idiopathic fibrotic disease. The increased activation of autophagy induces fibroblast-tomyofibroblast transition and promotes fibrotic tissue remodeling. Re-expression of MYST1 prevents aberrant autophagy, limits the profibrotic effects of TGF $\beta$ and ameliorates experimental fibrosis. Restoration of the epigenetic control of autophagy might thus ameliorate fibrotic tissue remodeling.

\section{Methods}

Patients. Dermal fibroblasts were isolated from skin biopsies of $23 \mathrm{SSc}$ patients and 12 age- and sex-matched healthy volunteers. All patients fulfilled the ACR/EULAR criteria for $\mathrm{SSc}^{72}$. Seventeen patients were female, six were male. The median age of SSc patients was 44 years (range: 19-61 years), and their median disease duration was 4 years (range: $0.5-8$ years). The median age was 51 years (range $40-68$ years).
Cell culture. Human dermal fibroblasts were isolated from twenty-three SSc patients and twelve age- and sex-matched healthy volunteers. Murine fibroblasts were isolated from skin biopsies of mice expressing two conditional alleles of Atg7 $\left(\right.$ Atg $\left.7^{\mathrm{fl} / \mathrm{fl}}\right)$ mice (kindly provided by Dr. Masaaki Komatsu, Tokyo Metropolitan Institute of Medical Science, Tokyo, Japan ${ }^{54}$ ). To delete Atg7, fibroblasts were infected with replication-incompetent adenovirus type 5 (Ad5) encoding for Cre (AdCre) recombinase. Human and murine fibroblasts were used in passages $4-8$ for all experiments. Two clones of Wi-26/SV-40 human lung fibroblasts with stable knockout of ATG7 (clones three and seven) were used in experiments to detect intracellular and secreted levels of type I collagen and TGF $\beta 1$. In selected experiments, fibroblasts were stimulated with recombinant TGF $\beta(10 \mathrm{ng} / \mathrm{ml})$ (PeproTech Hamburg, Germany), incubated with the Smad3 inhibitor SIS3 $(1 \mu \mathrm{M})$ (SigmaAldrich, Munich, Germany) and the autophagy inhibitor Chloroquine $(10 \mu \mathrm{M})$ (Biotrend, Cologne, Germany), 3MA (5 mM) (Sigma-Aldrich) and Bafilomycin A1 $(20 \mathrm{nM})$ (Sigma-Aldrich) for further analyses.

For viral infection experiments 80 ifu/cell of Ad5 were used. Ad5 encoding LacZ served as controls. Plasmid constructs were transfected into primary fibroblasts using the $4 \mathrm{D}-$ Nucleofector (Lonza, Cologne, Germany) ${ }^{73}$. The transfection efficiency was determined by co-transfection with $\mathrm{pSv}$ - $\beta$-galactosidase vectors (Promega, Mannheim, Germany). Gene silencing was achieved by transfection of 3 $\mu \mathrm{g}$ pre-designed siRNA duplexes against SMAD3 and MYST1 (Abgent, San Diego CA, USA) using the 4D-Nucleofector. Non-targeting siRNAs (nt siRNA) (Life Technologies, Darmstadt, Germany) served as controls

Generation of ATG7 knockout cells using CRISPR/Cas9. Human embryonic lung fibroblasts WI26/SV-40 were used to generate stable ATG7 knock-out clones Two independent clones (three and seven) were generated using the CRISPR/ Cas9 system ${ }^{74}$. In brief, double-stranded DNA oligonucleotides that encode guide RNAs (gRNAs) against ATG7 were cloned into the BbsI restriction sites of the pX459 vector. The gRNA oligonucleotides sequences used for the generation of the ATG7 KO lines were:

\section{hATG7-gRNA-ex3-s 5' caccgAACTCCAATGTTAAGCGAGC 3' \\ hATG7-gRNA-ex3-as 5' aaacGCTCGCTTAACATTGGAGTTc 3'}

Cells were transfected with the resulting vectors using XtremeGENE HP (Sigma Aldrich) according to the manufacturer's protocol and selected with puromycin $(2 \mu \mathrm{g} / \mathrm{ml})$ for 5 days. Single cell clones were picked using cloning cylinders (SigmaAldrich), clones deficient for the targeted protein were identified by immunoblot and knock-out clones were validated by genomic DNA sequencing using the following primers: hATG7-in2-s: 5' CCTGGCTGAGTCCCAGCTGTG 3' hATG7in3-as: 5' GAAGACACTGCAGAGACTAC 3'. The respective control cell line was generated by using the empty vector and following the same procedure described above for the knockout cell lines.

Mouse models of fibrosis. The functional role of the autophagy was investigated in the following mouse models of fibrosis: (i) In the model of bleomycin-induced dermal fibrosis, fibrosis was induced by local injections of bleomycin in C57BL/ 6NRj mice (Janvier Labs, Le Genest Saint Isle, France) for four weeks ${ }^{75,76}$. Subcutaneous injections of $0.9 \% \mathrm{NaCl}$, the solvent of bleomycin, served as control. (ii) In the T $\beta$ RIact model, injections of Ad5 encoding T $\beta$ RIact-induced localized skin fibrosis. Mice (C57BL/6NRj, Janvier Labs) injected with Ad5 encoding LacZ served as controls. $6.67 \times 10^{7}$ ifu of Ad5 were injected intracutaneously every 2 weeks and mice were sacrificed after 8 weeks 77,78 . (iii) In the model of bleomycin-induced pulmonary fibrosis, bleomycin was applied intratracheally in C57BL/6NRj mice (Janvier Labs) using a high pressure syringe (Penn-Century, Wyndmoor, PA, USA $)^{73,79}$. Instillation of equal volumes of $0.9 \% \mathrm{NaCl}$ served as controls. The outcome was evaluated after four weeks. (iv) In the model of a T $\beta$ RIact-induced pulmonary fibrosis, viral vectors (T $\beta$ RIact or LacZ) were instilled intratracheally every 2 weeks and mice (C57BL/6NRj, Janvier Labs) were sacrificed after eight weeks. To follow autophagic flux in vivo, $6.67 \times 10^{7}$ ifu of Ad5 encoding the 
a

\section{Cultured fibroblasts}

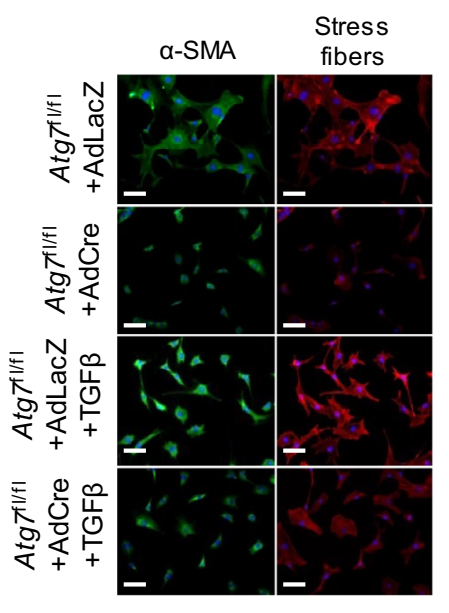

$\square \operatorname{Atg} 7^{\mathrm{fl} / f l}$ AdLacZ

$\square$ Atg $7^{\text {fl/fl }}$ AdCre

$\square$ Atg $^{\text {fl/ff }}$ AdLacZ+TGF $\beta$

$\square \operatorname{Atg}^{\mathrm{fl} / \mathrm{fl}} \mathrm{AdCre}+\mathrm{TGF} \beta$

$\alpha$-SMA

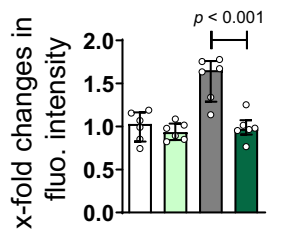

Stress fibers

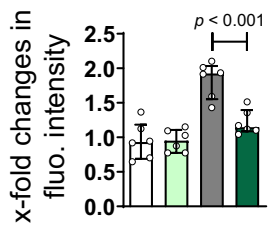

b

\section{Cultured fibroblasts}

$\square$ Atg $7^{f / / f l}$ AdLacZ $\square$ Atg $7^{\text {fl/fl }}$ AdLacZ+TGF $\beta$

$\square$ Atg $^{f / / f l}$ AdCre $\quad \square$ Atg $^{\text {fl/fil }}$ AdCre+TGF $\beta$
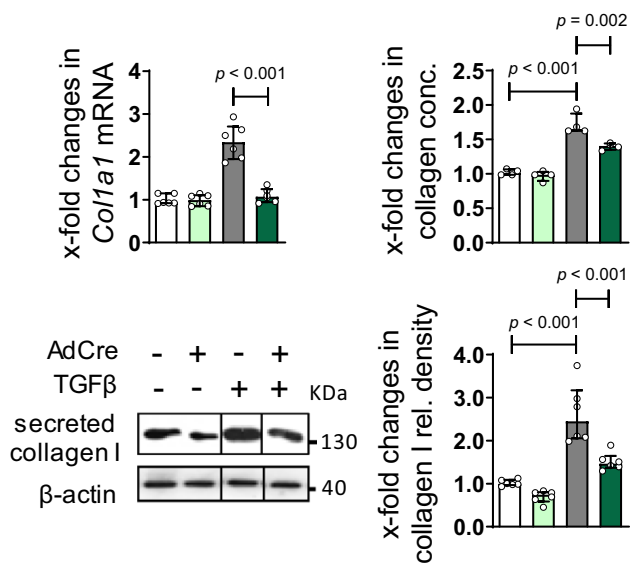

C

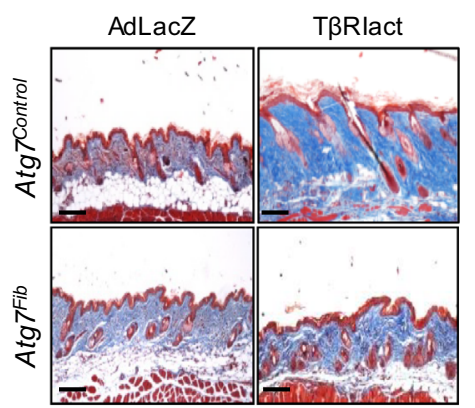
$\square$ Atg7 $7^{\text {Control }}$ AdLacZ
$\square$ Atg $7^{\text {Control }}$ TRlact
$\square$ Atg $^{\text {Fib }}$ AdLacZ

d
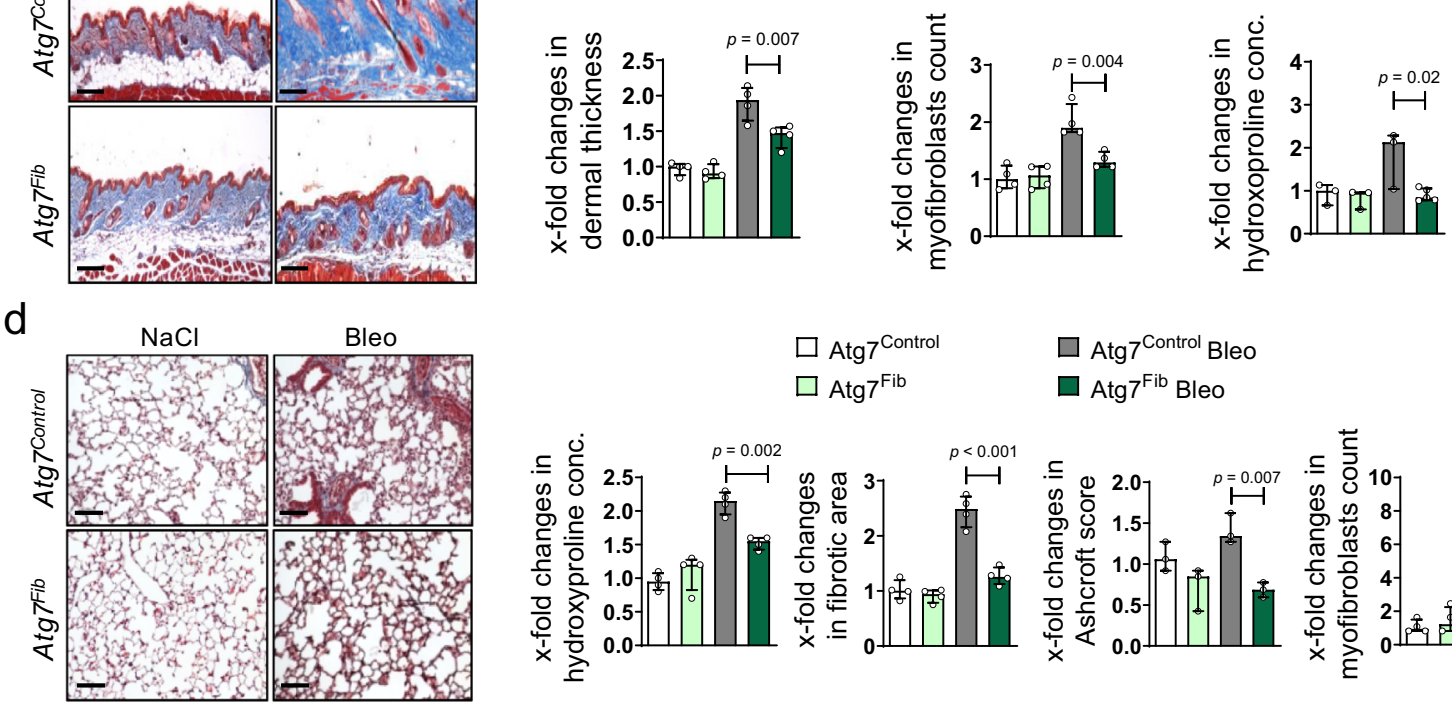

$$
\begin{aligned}
& \square \text { Atg } 7^{\text {Control Bleo }} \\
& \square \text { Atg } 7^{\text {Fib Bleo }}
\end{aligned}
$$

e
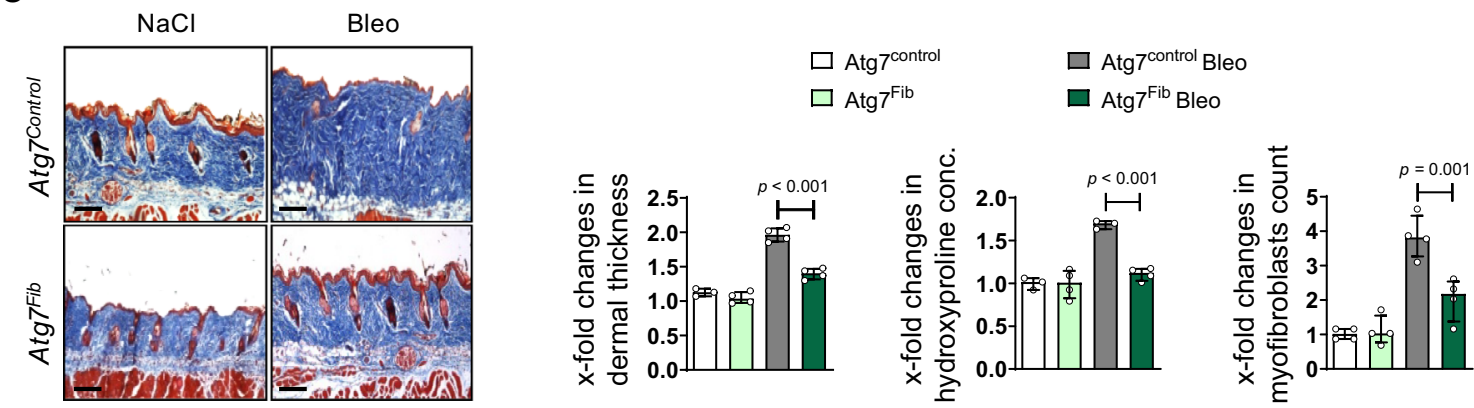

autophagy reporter EGFP/RFP-LC3 was injected intracutaneously (C57BL/6NRj, Janvier Labs) once per week for 4 weeks.

To inhibit TGF $\beta$ signaling in vivo, the ATP-competitive transforming growth factor $\beta$ receptor 1 (TGF $\beta \mathrm{RI}$ ) inhibitor 2-(5-Chloro-2-fluorophenyl)-4-((4-pyridyl) amino)pteridine SD208 (Tocris, Bristol, UK) was administered orally at a doses of $20 \mathrm{mg} / \mathrm{kg}$ twice a day for four weeks. Mice used in this experiment had a C57BL/ $6 \mathrm{NRj}$ background (Janvier Labs). All animals used in this study were maintained in laboratory animal housing facilities under follow conditions: Temperature: $20-24^{\circ}$
C; Humidity: $45-65 \%, 12 / 12 \mathrm{~h}$ light/dark cycle. Animals were 6-8 weeks old at the beginning of the experiments.

Plasmid and reporter constructs. The following expression and reporter plasmids were used: adenoviral expression constructs encodings murine Beclin1 (Vector Biolabs, Malvern, PA, USA), murine Myst1 (Applied Biological Materials, Richmond, Canada), In addition, we cloned the MYST1 promoter ( $-684 \mathrm{bp}$ to $+7 \mathrm{bp})$ 
Fig. 6 Knockout of Atg7 ameliorates fibroblast activation and experimental dermal and pulmonary fibrosis. a, b Cultured murine Atg7 knockout fibroblasts. a Expression of $\alpha \mathrm{SMA}$ and formation of stress fibers. Representative images and quantification $(n=6$ biological replicates per group). $\mathbf{b}$ mRNA levels of Co1la1 analyzed by qRT-PCR ( $n=6$ biological replicates per group), type I collagen protein as analyzed by western blot ( $n=6$ biological replicates per group) and total collagen analyzed by hydroxyproline assays ( $n=4$ biological replicates per group). Horizontal scale bar, $100 \mu$ m. c-e Mice with fibroblast-specific knockout of Atg7. c T $\beta$ Rlact-induced skin fibrosis: Representative trichrome stainings, dermal thickening, myofibroblast counts ( $n=4$ biological replicates per group) and hydroxyproline content ( $n=5$ biological replicates for Atgfib T $\beta$ Rlact group, $n=3$ other groups). d Experimental pulmonary fibrosis induced by intratracheal instillation of bleomycin: Representative trichrome stainings, hydroxyproline content, fibrotic area ( $n=4$ biological replicates per group), Ashcroft scores ( $n=3$ biological replicates per groups) and myofibroblast counts ( $n=4$ biological replicates per group). e Experimental dermal fibrosis induced by subcutaneous injections of bleomycin: Representative trichrome stainings, dermal thickening ( $n=3$ biological replicates for Atg7control and $n=4$ for other groups) myofibroblast counts ( $n=4$ biological replicates per group) and hydroxyproline content $(n=3$

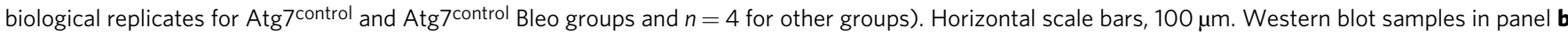
were run on the same gel. Images were cropped at the lines only for the purpose of this figure. All data are presented as median $\pm I Q R$. $p$-values were determined by ANOVA one-way with Tukey's multiple comparison post hoc test and are indicated in the figure. See source data for more detailed information. rel.: relative, Ad: adenovirus, fluo.: fluorescence, conc.: concentration. T $\beta$ Rlact: constitutively active TGF $\beta$ receptor type I, Bleo: bleomycin, Atg7control: littermate control mice Atg fib: fibroblast-specific Atg7 knockout mice.

into pGL3-basic luciferase reporter backbone (Catalog \#11979; Addgene, Watertown, MA, USA) (pGL3-MYST1-promoter) and mutated three predicted SBEs in the promoter sequence at residues -279 to -282 (SBE1), at residues -384 to -387 (SBE2) and at residues -574 to -577 (SBE3) either individually or in combination by exchanging GTCT to ATGT (pGL3-MYST1-ATGT) using the QuickChange Multi site-directed mutagenesis kit (Catalog\# 200514; Agilent Technologies, Santa Clara, CA, USA). Primers used for mutagenesis are listed in Supplementary Table 1. Overexpression of Myst 1 in murine skin and lungs was achieved by intracutaneous injection or intratracheal instillation of Ad5 encoding for Myst1 (Applied Biological Materials). Ad5 encoding LacZ served as controls. Autophagy was monitored as described using a reporter construct that encoded the rat LC3 cDNA fused to EGFP at the N-terminus and mRFP1 at the C-terminus of the insert $\left(\right.$ EGFP-LC3-RFP) ${ }^{80}$. The insert sequence was amplified from PMRX-IP-GFP-LC3$\mathrm{RFP}^{80}$ (plasmid \#84573; Addgene) and cloned in Ad5 using Adeno- $\mathrm{X}^{\mathrm{TM}}$ Adenoviral System 3 kit (Clontech, California, CA, USA). The FLAG-(5 A) construct was generated by exchanging the non-tagged sequence of the pcDNA4TO-hproTGF $\beta 1$ construct ${ }^{26}$ with a synthethic DNA (GeneART Strings, Thermo Fisher Scientific, Waltham, MA) sequence containing the FLAG-tagged growth factor sequence flanked by the sequence encoding the first 5 amino acids of the growth factor (aa $279-283)^{81}$ using the internal KpnI and the XhoI cloning site of the pcDNA4TOhproTGF $\beta 1$ construct.

Modulation of autophagy in experimental fibrosis. To selectively disable autophagy in fibroblasts, we crossbred mice carrying two conditional alleles of Atg7 $\left(\right.$ Atg $7^{f / / f l}$, provided by Dr. Masaaki Komatsu ${ }^{54}$ ) with Colla2;CreER mice ${ }^{8,82}$ to generate $\mathrm{Atg} 7^{\mathrm{fl} / \mathrm{fl}} \mathrm{X}$ Colla2;CreER mice. Cre-mediated recombination was induced by repeated intraperitoneal injections of $2 \mathrm{mg} / \mathrm{mL}$ tamoxifen over 5 days (Atg 7 Fib). Atg $7^{\mathrm{fl} / \mathrm{fl}} \mathrm{X}$ Colla2;CreER mice injected with corn oil served as controls (Atg $\left.7^{\mathrm{Control}}\right)$. All mice were on a C57BL/6 background.

To stimulate autophagy in vivo, $6.0 \times 10^{7}$ ifu of Ad5 encoding Beclin1 (Vector Biolabs) were injected intracutaneously or intratracheally, respectively. To knockdown Atg7 in vivo, we injected Atg7 siRNA or non-targeting siRNA (predesigned, Dharmacon, CA, USA) using the AteloGene in vivo siRNA/miRNA Transfection Kit (Reprocell, Glasgow, UK). One nmol of siRNA was injected subcutaneous once per week for three weeks. For pharmacological inhibition of autophagy in vivo, 3-methyladenine (3MA) (Sigma-Aldrich) was administered i.p. in a concentration of $15 \mathrm{mg} / \mathrm{kg}$ daily. Mice used in this experiment were on a C57BL/6NRj background (Janvier Labs).

Quantitative real-time-PCR. Gene expression was quantified by SYBR Green realtime PCR using the ABI Prism 7300 Sequence Detection System (Life Technologies). Samples without enzyme in the reverse transcription reaction (Non-RT controls) were used as negative controls. Unspecific signals caused by primer dimers were excluded by non-template controls and by dissociation curve analysis. Beta-actin was used to normalize the amounts of cDNA within each sample ${ }^{83}$. Primer sequences are listed in the Supplementary Table 2. qRT-PCRs were performed using StepOnePlus ${ }^{\mathrm{mt}}$ Real-Time PCR System and analyzed with StepOne software version 2.3 (Thermo Fisher Scientific).

Western blot analysis. Proteins were separated by sodium dodecyl sulfate polyacrylamide gel electrophoresis and transferred to a polyvinylidene difluoride membrane (PVDF). Membranes were incubated with primary antibodies listed in the Supplementary Table 3 and appropriate HRP-conjugated secondary antibodies (Dako, Glostrup, Denmark). Blots were visualized by ECL. $\beta$-actin was used as loading control (see Supplementary Table 3 for more information about antibodies). Western blot images were detected using ChemiDoc MP Imaging System (BioRad, Hercules, CA, USA) or X-ray film (GE Healthycare, Chicago, IL, USA).
Blots were quantified using the ImageJ Software version 1.41 (National Institutes of Health, Bethesda, MD, USA) or Image Lab version 6.0.0 (BioRad).

SILAC-based quantitative proteomics. For the quantitative analysis of the ATG7-dependent secretome, wild-type and ATG7 knock-out cells were pulselabeled at $70 \%$ confluency. In brief, cells were pre-incubated with Met/Arg/Lys-free DMEM/F12 (Athena Enzyme Systems, Baltimore, MD, USA), supplemented with $1 \mathrm{mM}$ pyruvate, $200 \mathrm{mg} / \mathrm{l} \mathrm{L}$-proline and dialyzed FCS (SILAC medium) for $30 \mathrm{~min}$, to deplete intracellular Arg, Lys, and Met. Cells were then incubated for $24 \mathrm{~h}$ with $100 \mu \mathrm{M}$ of the azide group-containing methionine analog azidohomoalanine (AnaSpec, Seraing, Belgium) in SILAC medium either supplemented with medium $\left(146 \mu \mathrm{g} / \mathrm{ml} \mathrm{Lys}-4\left({ }^{2} \mathrm{H}\right), 84 \mu \mathrm{g} / \mathrm{ml}\right.$ Arg-6 $\left.\left({ }^{13} \mathrm{C}_{6}\right)\right)$ or heavy isotopes $(146 \mu \mathrm{g} / \mathrm{ml}$ Lys-8 $\left.\left.{ }^{13} \mathrm{C}_{6},{ }^{15} \mathrm{~N}_{2}\right) / 84 \mu \mathrm{g} / \mathrm{ml} \mathrm{Arg}-10\left({ }^{13} \mathrm{C}_{6},{ }^{15} \mathrm{~N}_{4}\right)\right)$. All isotope-labeled amino acids were purchased from Silantes (Munich, Germany). Supernatants of wildtype heavy isotope-labeled and knock-out medium isotope-labeled cultures were combined, dead cells were removed by centrifugation $\left(5 \mathrm{~min}, 1000 \mathrm{xg}, 4^{\circ} \mathrm{C}\right)$, and proteins were concentrated to approximately $250 \mu$ l, using Amicon Ultra-15 Centrifugal Filter Unit $3 \mathrm{kDa}$ cutoff concentrator tubes (Sigma Aldrich). Samples from the inverse combination (wild-type medium isotope-labeled and knock-out heavy isotope-labeled cultures) were also prepared to control for labeling bias. Cells were lysed in $850 \mu \mathrm{l}$ of urea lysis buffer provided with the Click Chemistry Capture kit (Jena Bioscience, Jena, Germany), according to the manufacturer's protocol. Supernatants and cell lysates were individually covalently coupled to $200 \mu \mathrm{l}$ prewashed alkyne agarose (Jena Bioscience) and rotated overnight at room temperature. Alkyne agarose-bound samples were spun down $\left(5 \mathrm{~min}, 2000 \mathrm{xg}, 4^{\circ} \mathrm{C}\right)$, washed once with $1 \mathrm{ml}$ HPLC water, reduced with $1 \mathrm{mM}$ DTT, alkylated with 40 $\mathrm{mM}$ chloroacetamide, transferred to columns (supplied with the Click Chemistry Capture kit) and extensively washed sequentially with SDS wash buffer (supplied with Click Chemistry Capture kit), $100 \mathrm{mM}$ Tris- $\mathrm{HCl}$, pH 8.0/8 M urea buffer, 20\% isopropanol, and $20 \%$ acetonitrile $(20 \mathrm{ml}$ each). Proteins were then digested overnight with $1 \mu \mathrm{g}$ trypsin (Serva, Heidelberg, Germany) and $0.5 \mu \mathrm{g}$ LysC (FUJIFILM Wako Chemicals, Neuss, Germany) in a heated shaker $\left(37^{\circ} \mathrm{C}\right.$, $800 \mathrm{rpm}$ ). Digestion was stopped by adding $0.5 \%$ formic acid to the reaction, and the peptides subjected to StageTip purification using styrene-divinylbenzene reverse-phase sulfonate (SDB-RPS) StageTips with two layers of SDB-RPS discs before injection into the mass spectrometer.

Mass spectrometry analysis. Proteomic analysis was performed using an Easy nLC 1000 UHPLC coupled to a QExactive Plus mass spectrometer (Thermo Fisher Scientific). Peptides were resuspended in Solvent A, picked up with an autosampler and loaded onto in-house made $50 \mathrm{~cm}$ fused silica columns (internal diameter 75 $\mu \mathrm{m}$, packed with C18 Poroshell beads, $2.7 \mu \mathrm{m}$, Agilent) at a flow rate of $0.75 \mu \mathrm{l} / \mathrm{min}$. Eluted peptides were sprayed into the heated transfer capillary of the mass spectrometer using a nano-electrospray ion source (Thermo Fisher Scientific). The mass spectrometer was operated in a data-dependent mode, where the Orbitrap acquired full MS scans $(300-1750 \mathrm{~m} / \mathrm{z})$ at a resolution (R) of 70,000 with an automated gain control (AGC) target of $3 \times 10^{6}$ ions collected within $20 \mathrm{~ms}$. The dynamic exclusion time was set to $20 \mathrm{~s}$. From the full MS scan, the 10 most intense peaks $(z \geq 2)$ were fragmented in the high-energy collision-induced dissociation (HCD) cell. Collisional energy, ion target and maximum injection time were adapted for the different input samples. The raw files were processed using MaxQuant software v1.5.3.8 (Max-Planck-Institute of Biochemistry, Martinsried, Germany) and its implemented Andromeda search engine ${ }^{84}$. Parameters were set to default values and SILAC labels were included as described in the proteomics section. Settings and parameters used for the mass spectrometry analysis are described in the Supplementary Table 4. 
a

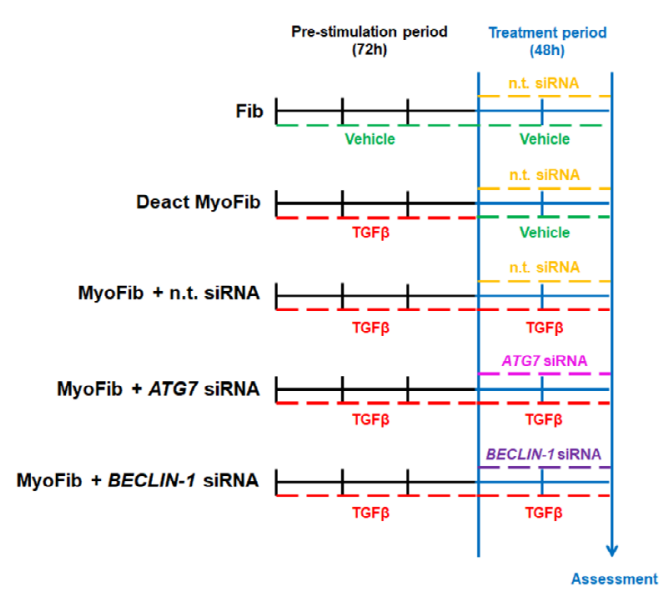

b
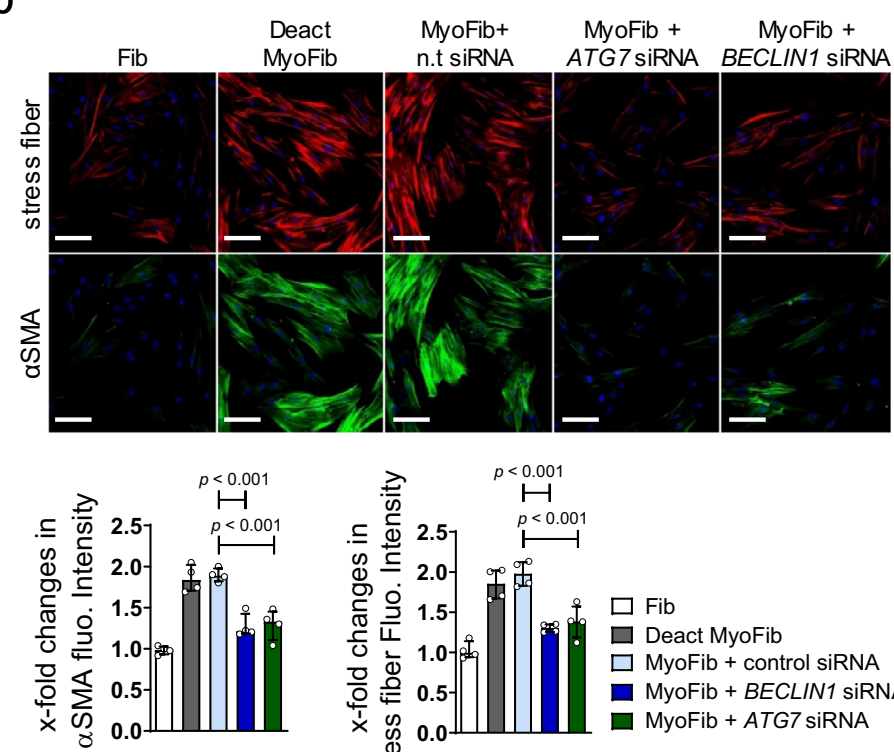

C

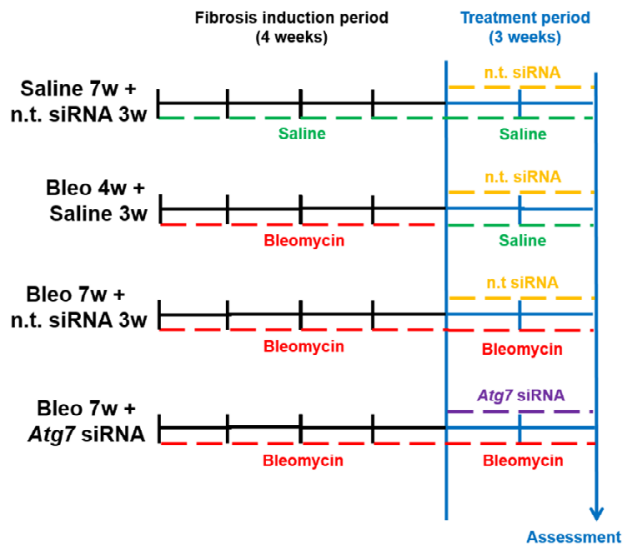

e

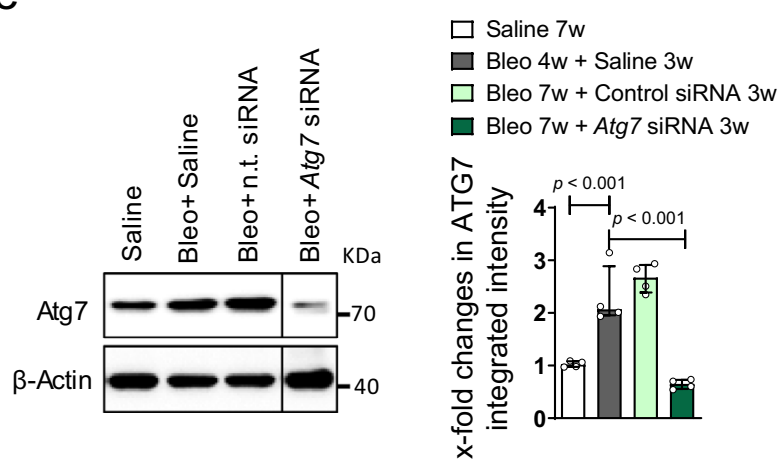

d
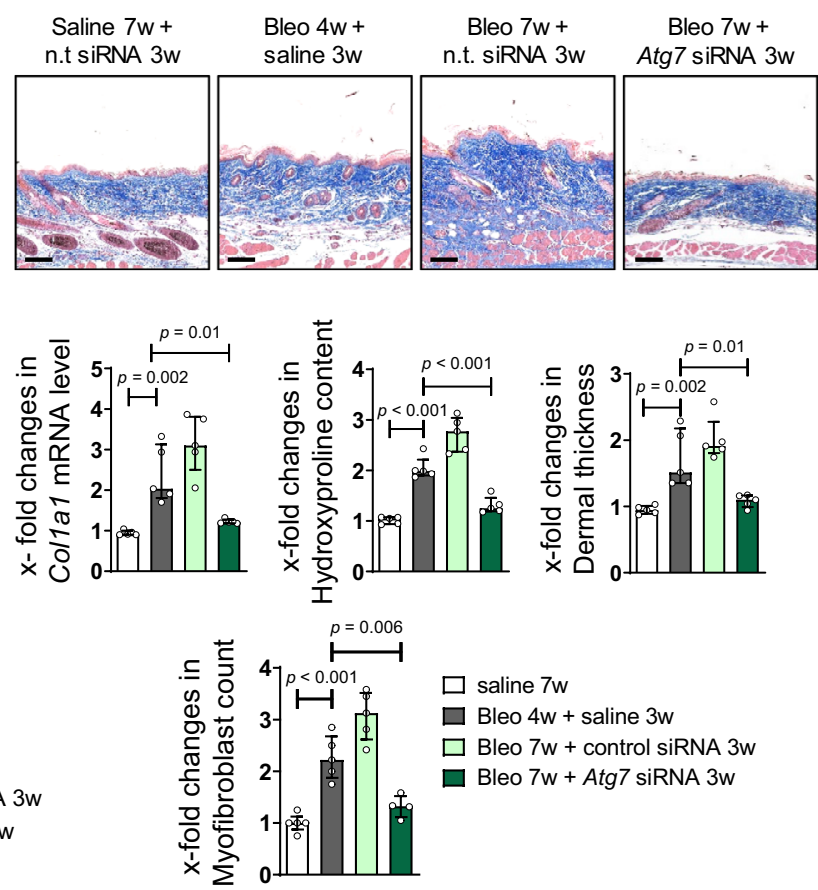

$\square$ saline $7 \mathrm{w}$

$\square$ Bleo $4 w+$ saline $3 w$

$\square$ Bleo $7 w+$ control siRNA 3w

$\square$ Bleo 7w + Atg7 siRNA 3w
Quantification of active TGF $\beta$ by mink lung cell (MLC) assay. The level of active TGF $\beta$ was evaluated by MLC transfected with a fused sequence of firefly luciferase and PAI-1 promoter (kindly provided by Daniel Rifkin ${ }^{85}$ ). In all, $4 \times 10^{4}$ MLCs were plated into a 96-well plate and incubated overnight at $37^{\circ} \mathrm{C}$. On the next day, $100 \mu \mathrm{l}$ of fibroblast-conditioned medium or $20 \mu \mathrm{l}$ of diluted murine skin lysates (100-300 $\mu$ g protein diluted in $500 \mu \mathrm{l}$ DMEM medium with $0.1 \%$ bovine serum albumin (BSA)) were added to the medium of pre-plated MLCs. After $20-24 \mathrm{~h}$ of stimulation, luciferase reporter activity was determined by using a microplate luminometer Centro LB 960 (Berthold Technologies, Bad Herrenalb, Germany). The value of luminescence was further converted into concentration by using a standard curve with a series dilution of recombinant TGF $\beta^{86}$.

Reporter assays. NIH3T3 mouse embryonic fibroblasts were transfected with different luciferase reporter constructs or $\mathrm{pSv}-\beta$-galactosidase $(\beta$-gal) using FuGENE HD Transfection Reagent (Promega) or infected with Ad5 luciferase 
Fig. 7 Inhibition of autophagy deactivates myofibroblasts and induces regression of fibrosis. a, b Knockdown of ATG7 and BECLIN-1 in human fibroblasts. a Schematic illustration of the experimental procedure. b Expression of $\alpha \mathrm{SMA}$ (green) and formation of stress fibers (red), costained with DAPI (blue). Representative images and quantification of $\alpha \mathrm{SMA}$ and Stress fibers fluorescence intensity ( $n=4$ biological replicates per group). Horizontal scale bar, $50 \mu \mathrm{m} . \mathbf{c}$, d Effects of Atg7 knockdown in a model of established bleomycin-induced skin fibrosis. $\mathbf{c}$ Schematic illustration of the experimental procedure. d Representative trichrome stainings, Col1a1 mRNA, hydroxyproline content, dermal thickness $(n=5$ biological replicates per group) and myofibroblast counts ( $n=4$ biological replicates for Bleo $7 w+$ Atg7 siRNA $3 w$ group and $n=5$ for other groups). e Representative western blot and quantification of ATG7 protein levels ( $n=4$ biological replicates per group). Horizontal scale bar, $100 \mu \mathrm{m}$. All data are presented as median \pm IQR. $p$-values were determined by ANOVA one-way with Tukey's multiple comparison post hoc test and are indicated in the figure. See source data for more detailed information. Fluo.: fluorescence, Bleo: bleomycin. Fib: fibroblasts, Deact MyoFib: deactivated myofibroblasts, w: weeks. Western blot samples in panel d were run on the same gel. Images were cropped at the lines only for the purpose of this figure.

reporter constructs. Luciferase activity was normalized for transfection efficiency to internal pSv- $\beta$-galactosidase. Luciferase activities were determined using a microplate luminometer Centro LB 960 (Berthold Technologies) and data was collected using Step 7 MicroWin v4.0 Sp9 software (Labsis Laborsysteme, NeunkirchenSeelscheid, Germany). To monitor the effects of TGF $\beta$ on autophagy in vitro, cells were infected with Ad5 encoding EGFP-LC3-RFP (described in the section plasmid and reporter constructs), an autophagy activity reporter. After infection, cells were incubated with different inhibitors: Vorinostat $(1 \mu \mathrm{M})$, Trichostatin $\mathrm{A}(0.4 \mu \mathrm{M})$ (both Selleckchem, Houston, TX, USA), 5-Aza-2'-deoxycytidine (10 $\mu \mathrm{M}$, SigmaAldrich) and 3-deazaneplanocin A (10 $\mu \mathrm{M}$, Tocris). In addition, Myst1 was knockdown using siRNA or overexpressed using adenoviral vectors. Fluorescence was detected by high-throughput real-time imaging technology using IncuCyte Live-Cell Analysis System (Essen Bioscience, Royston Hertfordshire, UK). Data was collected by Incucyte ${ }^{\circledast}$ S3 Software V2018A (Essen Bioscience).

Immunohistochemistry and immunofluorescence staining. Formalin-fixed, paraffin-embedded skin sections or cultured cells fixed with $4 \%$ PFA and permeabilized with $0.25 \%$ Triton X100 were stained with appropriate primary antibodies (see Supplementary Table 3). HRP-conjugated or Alexa Fluor antibodies (Life Technologies) were used as secondary antibodies. Isotype control antibodies were used as controls. Stress fibers were visualized with rhodamine-conjugated phalloidin (Sigma-Aldrich) (see Supplementary Table 3 for more information). In addition, cell nuclei were stained using DAPI (Santa Cruz Biotechnology, Heidelberg, Germany). Images were captured using a Nikon Eclipse 80i fluorescence microscope and data was collected using NIS-Elements BR version 5.20.01 software (Nikon, Badhoevedorp, Netherlands). Samples of mice tissue injected with Ad5 encoding EGFP-LC3-RFP (autophagy reporter) were immunostained for EGFP using polyclonal goat-anti-GFP antibody (Abcam, Cambridge, UK) and RFP using monoclonal rabbit anti-RFP (Abcam) and analyzed by fluorescence microscopy using the same microscope described above (see Supplementary Table 3 for more information about antibodies used). Fluorescence intensity was quantified using ImageJ/Fiji software (National Institutes of Health).

Costainings of LC3B and P62 with LAMP2 (see Supplementary Table 3) in skin tissue were analyzed by confocal microscopy using the Leica SP5 II microscope (Leica Microsystems, Wetzlar, Germany) and data was collected using Leica Application Suite X version 4.12.0.86 (Leica Mycrosystems). Confocal images were deconvoluted by using DeconvolutionLab2 (EPFL, Lausanne, Switzerland) based on corresponding optical parameters ${ }^{87}$.

Quantification was performed with ImageJ software (version 1.41; National Institutes of Health) as follows: a density threshold was set to quantify the positive staining by using the respective negative controls. The threshold was selected to exclude unspecific background staining. The same thresholds and system settings were used for all slides. The number of pixels falling within the threshold, indicating the quantity of positive staining, was recorded for each area.

Apoptosis detection. To monitor apoptosis in vitro, we used Caspase- 3/7 Green Apoptosis Reagent (Essen Bioscience) according to the recommendations of the manufacturer. Fluorescence was detected by high-throughput real-time imaging technology using IncuCyte Live-Cell Analysis System (Essen Bioscience). Data was collected by Incucyte ${ }^{\circledast}$ S3 Software V2018A (Essen Bioscience). As positive control for apoptosis, cells were treated with $0.1 \mu \mathrm{M}$ staurosporine (Tocris). In vivo, apoptosis was detected using TACS in situ apoptosis detection kit (Trevigen, Gaithersburg, MD, USA). Images were captured using the fluorescence microscopy Nikon Eclipse 80i microscope and the software NIS-Elements BR version 5.20.01 (Nikon).

Myofibroblast re-differentiation assays. Human fibroblasts were cultured in 96well plates. After $24 \mathrm{~h}$, we differentiated fibroblasts into myofibroblasts by incubation with TGF $\beta$ for three days, with re-stimulation with TGF $\beta$ every twenty-four hours. We then treated the cells with CQ $(10 \mu \mathrm{M}$, Biotrend) or 3-MA $(5 \mathrm{mM}$, Sigma-Aldrich). Alternatively, we knocked down ATG7 and BECLIN1 (50 nM) (pre-designed, Invitrogen, CA, USA), via siRNA transfection using Viromer Blue transfection reagent (OriGene Technologies, Rockville, MD, USA), and continued the stimulation with recombinant TGF $\beta$ for two additional days. The control group (Fib) was pre-treated with vehicle for three days, then transfected with nontargeting siRNA and treated with vehicle for additional two days. The deactivated myofibroblast group (deact MyoFib) was pre-treated with TGF $\beta$ for three days, then transfected with non-targeting siRNA and treated with vehicle for two days. Afterwards, cells were fixed with 4\% PFA and immunostained for stress fibers and aSMA as described above (immunohistochemistry and immunofluorescence staining section). Images were captured using CellInsight CX5 High-Content Screening (HCS) Platform (Thermo Fisher Scientific; software HCS Navigator Version 6.6.1). Data were analyzed using HCS Studio Cell Analysis Software 6.6.1 (Thermo Fisher Scientific)

Extracellular matrix Immunofluorescence staining. Fibroblasts were platted in a 96-well, black-walled imaging plates (BD Biosciences, NJ, USA) at a density of $4 \times$ $10^{3}$ cells/well. Fibroblasts were incubated for 5 days with or without treatment. Afterwards, plates were washed with PBS and cells were removed with $0.25 \mathrm{M}$ ammonium hydroxide in $25 \mathrm{mM}$ Tris for $15 \mathrm{~min}$ at $37^{\circ} \mathrm{C}$. The plate with the remaining extracellular matrix (ECM) was washed three times with PBS and fixed with $100 \%$ methanol for $30 \mathrm{~min}$ at $-20^{\circ} \mathrm{C}$ and finally washed three more times with PBS. ECM was immunostained with monoclonal mouse anti-Fibronectin antibodies conjugated with Alexa Fluor 488 (eBiosciences, Frankfurt am Main, Germany), polyclonal rabbit anti-Collagen Type I antibodies (Merck Millipore, Darmstadt, Germany) and polyclonal rabbit anti-Collagen Type III antibodies (Merck Millipore). See Supplementary Table 3 for more information about antibodies used in this experiment. Samples were analyzed using CX5-based automated quantification of extracellular matrix (Thermo Fisher Scientific; software HCS Navigator Version 6.6.1). Data were analyzed using HCS Studio Cell Analysis Software 6.6.1 (Thermo Fisher Scientific).

Transmission electron microscopy. For transmission electron microscopy, cells were harvested and washed with $0.1 \mathrm{M}$ phosphate buffer (PB), followed by fixing with $2 \%$ glutaraldehyde and $2 \%$ paraformaldehyde in $0.1 \mathrm{M} \mathrm{PB}$ for $3 \mathrm{~min}$, and postfixation with $1 \%$ osmic acid for $1 \mathrm{~h}$. The samples were then dehydrated in graded ethanol, washed with propylene oxide, and embedded in epoxy resin. Samithin and Ultrathin sections cut in a Reichert ultramicrotome were stained with lead citrate and uranyl acetate and were examined in a HITACHI H-7500 TEM at $100 \mathrm{kV}$. Images were captured with AMT XR-16 $16 \mathrm{mp}$ high-resolution CCD camera system in combination with the AMT Capture Engine, v602.600.51 (Woburn, MA, USA).

Chromatin immunoprecipitation (ChIP) assays. ChIP assays were performed using the ChIP-IT Express Kit (Active Motif, Carlsbad, CA, USA). Sonicated chromatin extracts were incubated with antibodies against SMAD3 (Cell signaling) or normal rabbit IgG antibodies (Santa Cruz Biotechnology). See Supplementary Table 3 for more information about the antibodies used. Bound DNA sequences were determined by quantitative real-time PCR. Primer sequences are listed in the Supplementary Table 5.

Evaluation of experimental fibrosis. The extent of fibrosis was analyzed using histological, biochemical, immunohistochemical and mRNA readouts. Histologic readouts included quantification of the dermal thickness as analyzed by measuring the distance between the epidermal-dermal junction and the dermal-subcutaneous fat junction on HE stained sections at eight sites at 100-fold magnification, evaluation of the fibrotic area as percent of total lung area in Sirius Red stained sections; quantification of pulmonary changes using the Ashcroft score and direct visualization of collagen by trichrome staining $83,88,89$. The total collagen content was analyzed biochemically using hydroxyproline assays and by evaluation of Colla1 mRNA. In addition, myofibroblasts were identified immunohistochemically as $\alpha$-smooth muscle actin positive fibroblasts at four different sites in a tissue ${ }^{90,91}$. To quantify the pulmonary fibrosis in animal models, we analyzed histological sections and classified the changes with scores from 0 to 8 according to the Ashcroft scale ${ }^{92,93}$. Images were captured using Nikon Eclipse 80i microscope in combination with NIS-Elements BR 
a

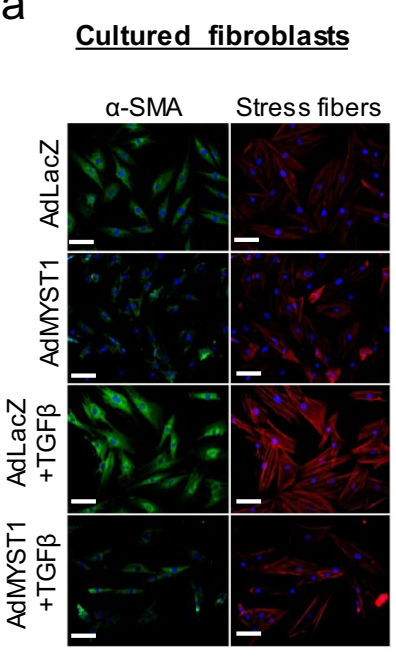

$\square$ AdLacZ

$\square$ AdMYST1

$\square$ AdLacZ+TGF $\beta$

$\square$ AdMYST1+TGF $\beta$

stress fibers
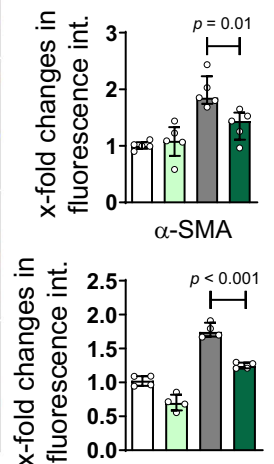

b

\section{Cultured fibroblasts}

\section{$\square$ AdLacZ $\square$ AdLacZ+TGF $\beta$ \\ $\square$ AdMYST1 $\square$ AdMYST1+TGF $\beta$}
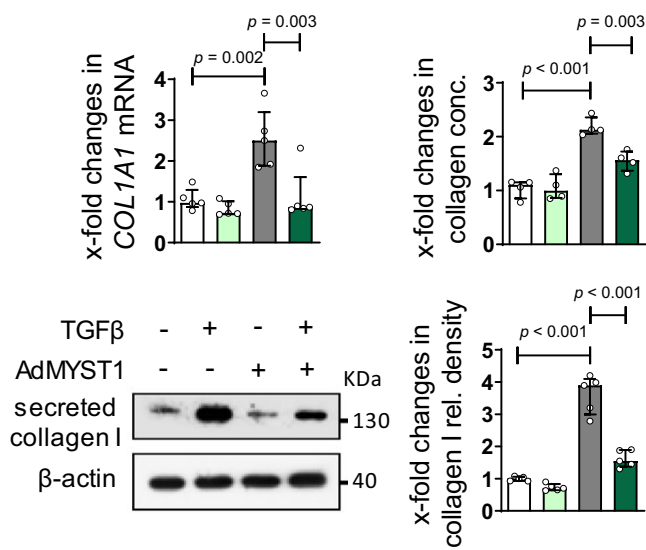

C
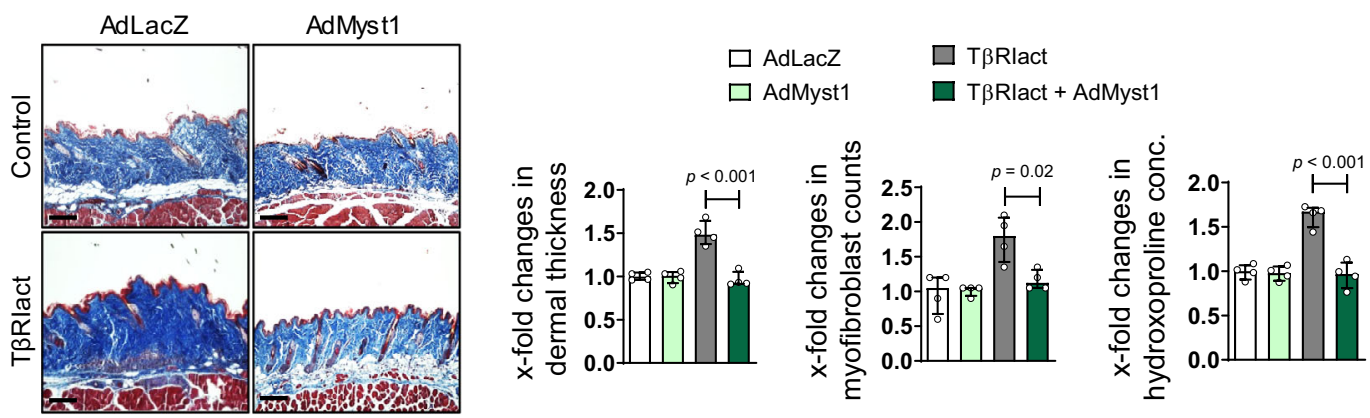

d
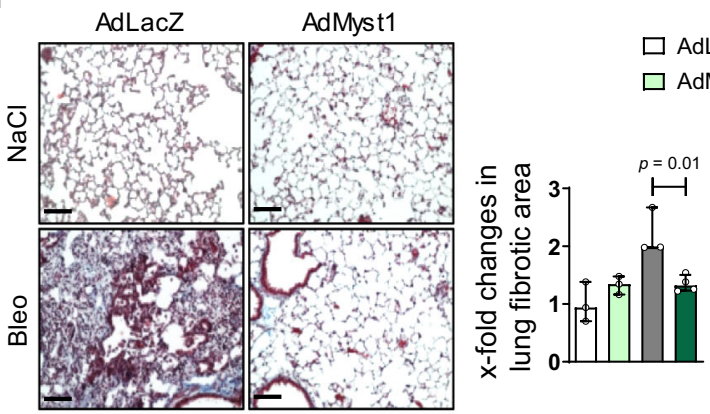

$\square$ AdLacZ $+\mathrm{NaCl}$

$\square$ AdLacZ + Bleo

AdMYST1 + $\mathrm{NaCl}$

$\square$ AdMYST1 + Bleo

e
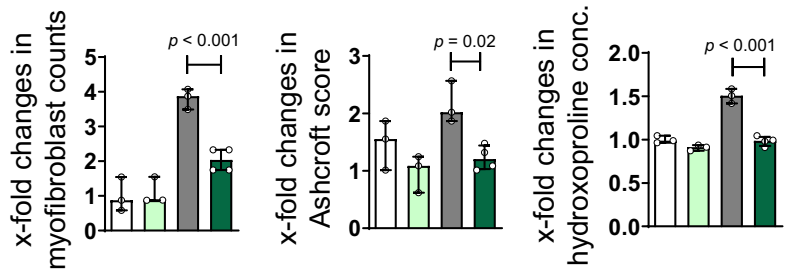

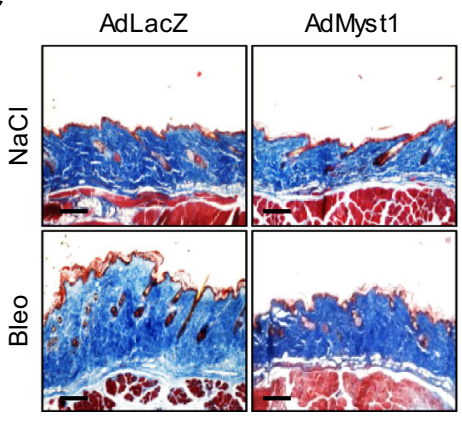

AdLacZ AdMyst1 $\square$ AdLacZ + NaCl $\square$ AdLacZ + Bleo

$\square$ AdMYST1 + NaCl $\square$ AdMYST1 + Bleo version 5.20.01 software (Nikon) or Nano Zoomer S60 slide scanner using NDP.scan 3.3 software for data collection and NDP.view 2 software for analysis (Hamamatsu Photonics, Hamamatsu City, Japan).

Statistics and reproducibility. The sample size was estimated based on previous experiments. No statistical method was used to predetermine sample size. Experiments and quantifications were not done in a blinded fashion. There were no exclusion criteria for the human and animal experiments. Mice were stratified according to sex and then randomized into the different treatment groups. Cells from human donors were also randomized. Unless otherwise noted, each experiment was repeated at least three times independently. Data shown in column graphs are presented as median \pm interquartile range (IQR) as indicated in the figure legends, and individual data points are plotted. Differences between the groups were tested for their statistical significance by Mann-Whitney $U$ nonparametric test for non-related samples and by ANOVA one-way with Sidak's 
Fig. 8 Re-expression of MYST1 inhibits TGF $\beta$-dependent fibroblast activation and prevents experimental fibrosis. a, b Overexpression of MYST1 in cultured human dermal fibroblasts: a Expression of $\alpha \mathrm{SMA}(n=4$ biological replicates per group) and formation of stress fibers ( $n=5$ biological replicates per group). Representative images and quantification. Horizontal scale bar, $100 \mu \mathrm{m}$. b mRNA levels of COL1A1 analyzed by qRT-PCR ( $n=5$ biological replicates per group), total collagen as analyzed by hydroxyproline assays ( $n=4$ biological replicates per group) and type I collagen protein as analyzed by western blot ( $n=5$ biological replicates per group). c-e Adenoviral overexpression of MYST1 in murine models. c T $\beta$ Rlact-induced skin fibrosis: Representative trichrome stainings for, dermal thickening, myofibroblast counts and hydroxyproline content $(n=4$ biological replicates per group). $\mathbf{d}$ Bleomycin-induced pulmonary fibrosis: Representative Trichrome stainings, fibrotic area, myofibroblast counts, Ashcroft scores and hydroxyproline content ( $n=4$ biological replicates for the group AdMyst1 treated with TGF $\beta$ and $n=3$ for other groups). e Bleomycin-induced dermal fibrosis: Representative trichrome stainings shown, dermal thickening, myofibroblast counts and hydroxyproline content $(n=4$ biological replicates for bleomycin treated groups and $n=3$ for saline treated groups). Horizontal scale bars, $100 \mu \mathrm{m}$. All data are presented as median \pm IQR. $p$-values were determined by ANOVA one-way with Tukey's multiple comparison post hoc test and are indicated in the figure. See source data for more detailed information. rel.: relative, Ad: adenovirus, conc.: concentration, int.: intensity, T $\beta$ Rlact: constitutively active TGF $\beta$ receptor type I, Bleo: bleomycin.

multiple comparisons test or Tukey's multiple comparison post hoc test for comparisons involving more than two groups. Information about $p$-values and statistical tests used in this article can be found in the figure legends and in the source data file. Statistic was analyzed using GraphPad prism 8 version 8.3.0 (GraphPad Software, San Diego, CA, USA). For SILAC experiments a one-sample $t$-test was performed (Value $=0, S 0=0.1$, side $=$ both) and $-\log [p$-value $]$ was calculated using Perseus version 1.6.5

Study approval. The human studies were approved by the Ethical committee of the Medical Faculty of the University of Erlangen-Nuremberg. All patients and controls signed a consent form approved by the review board of the institution mentioned above. All human studies were performed in compliance with the relevant ethical regulations of the Medical Faculty of the University of ErlangenNuremberg. All animal experiments were approved by the governments of Mittelfranken or Unterfranken, Germany. All animal experiments were performed in compliance with the relevant ethical regulations of the governments of Mittelfranken or Unterfranken, Germany.

Reporting summary. Further information on research design is available in the Nature Research Reporting Summary linked to this article.

\section{Data availability}

All data generated or analyzed during this study are included in this article and its supplementary information files. Source data are provided with this article. Uncropped western blot images are provided in the source data file. Additional detailed information is available from the corresponding author on reasonable request. The proteomics data are available via the PRIDE database with the dataset identifier PXD025001. As long as the full PRIDE dataset has not been publicly released, login credentials to the PRIDE reviewer account will be provided upon reasonable request (JN). Source data are provided with this paper.

Received: 23 September 2019; Accepted: 29 April 2021; Published online: 20 July 2021

\section{References}

1. Wynn, T. A. Cellular and molecular mechanisms of fibrosis. J. Pathol. 214, 199-210 (2008).

2. Gurtner, G. C., Werner, S., Barrandon, Y. \& Longaker, M. T. Wound repair and regeneration. Nature 453, 314-321 (2008).

3. Friedman, S. L., Sheppard, D., Duffield, J. S. \& Violette, S. Therapy for fibrotic diseases: nearing the starting line. Sci. Transl. Med. 5, 167sr161 (2013).

4. Elhai, M., Avouac, J., Kahan, A. \& Allanore, Y. Systemic sclerosis: recent insights. Jt. Bone Spine.: Rev. du Rhum. 82, 148-153 (2015).

5. Distler, J. H. et al. Review: frontiers of antifibrotic therapy in systemic sclerosis. Arthritis Rheumatol. 69, 257-267 (2017).

6. Gyorfi, A. H., Matei, A. E. \& Distler, J. H. W. Targeting TGF-beta signaling for the treatment of fibrosis. Matrix biology: journal of the International Society for. Matrix Biol. 68-69, 8-27 (2018).

7. Verrecchia, F. \& Mauviel, A. Transforming growth factor-beta and fibrosis. World J. Gastroenterol. 13, 3056-3062 (2007).

8. Sonnylal, S. et al. Postnatal induction of transforming growth factor beta signaling in fibroblasts of mice recapitulates clinical, histologic, and biochemical features of scleroderma. Arthritis Rheum. 56, 334-344 (2007).

9. Zeisberg, E. M. \& Zeisberg, M. The role of promoter hypermethylation in fibroblast activation and fibrogenesis. J. Pathol. 229, 264-273 (2013).
10. Mann, J. \& Mann, D. A. Epigenetic regulation of wound healing and fibrosis. Curr. Opin. Rheumatol. 25, 101-107 (2013).

11. Hardy, T. \& Mann, D. A. Epigenetics in liver disease: from biology to therapeutics. Gut 65, 1895-1905 (2016).

12. O'Reilly, S. Epigenetics in fibrosis. Molecular aspects of medicine. Gut 65, 1895-1905 (2016).

13. Shin, J. Y. et al. Epigenetic activation and memory at a TGFB2 enhancer in systemic sclerosis. Sci. Transl. Med. 11, eaaw0790 (2019).

14. Bechtel, W. et al. Methylation determines fibroblast activation and fibrogenesis in the kidney. Nat. Med. 16, 544-550 (2010).

15. Wang, Y., Fan, P. S. \& Kahaleh, B. Association between enhanced type I collagen expression and epigenetic repression of the FLI1 gene in scleroderma fibroblasts. Arthritis Rheum. 54, 2271-2279 (2006).

16. Noda, S. et al. Simultaneous downregulation of KLF5 and Fli1 is a key feature underlying systemic sclerosis. Nat. Commun. 5, 5797 (2014).

17. Sanders, Y. Y. et al. Histone deacetylase inhibition promotes fibroblast apoptosis and ameliorates pulmonary fibrosis in mice. Eur. Respiratory J. $\mathbf{4 3}$ 1448-1458 (2014)

18. Pang, M. \& Zhuang, S. Histone deacetylase: a potential therapeutic target for fibrotic disorders. J. Pharmacol. Exp. Ther. 335, 266-272 (2010).

19. Mann, J. et al. MeCP2 controls an epigenetic pathway that promotes myofibroblast transdifferentiation and fibrosis. Gastroenterology 138, 705-714 (2010). 714 e701-704.

20. Zeybel, M. et al. A proof-of-concept for epigenetic therapy of tissue fibrosis: inhibition of liver fibrosis progression by 3-deazaneplanocin A. Mol. Ther: J. Am. Soc. Gene Ther. 25, 218-231 (2017).

21. Zeybel, M. et al. Multigenerational epigenetic adaptation of the hepatic wound-healing response. Nat. Med. 18, 1369-1377 (2012).

22. Wang, C. W. \& Klionsky, D. J. The molecular mechanism of autophagy. Mol. Med. 9, 65-76 (2003).

23. Deretic, V., Jiang, S. \& Dupont, N. Autophagy intersections with conventional and unconventional secretion in tissue development, remodeling and inflammation. Trends Cell Biol. 22, 397-406 (2012).

24. Ponpuak, M. et al. Secretory autophagy. Curr. Opin. Cell Biol. 35, 106-116 (2015).

25. Guo, H. et al. Atg5 disassociates the $\mathrm{V}(1) \mathrm{V}(0)$-ATPase to promote exosome production and tumor metastasis independent of canonical macroautophagy. Dev. Cell 43, 716-730.e717 (2017)

26. Nuchel, J. et al. TGFB1 is secreted through an unconventional pathway dependent on the autophagic machinery and cytoskeletal regulators. Autophagy 14, 465-486 (2018).

27. Leidal, A. M. et al. The LC3-conjugation machinery specifies the loading of RNA-binding proteins into extracellular vesicles. Nat. Cell Biol. 22, 187-199 (2020).

28. Narita, M. et al. Spatial coupling of mTOR and autophagy augments secretory phenotypes. Science 332, 966-970 (2011).

29. Gee, H. Y., Noh, S. H., Tang, B. L., Kim, K. H. \& Lee, M. G. Rescue of $\Delta$ F508CFTR trafficking via a GRASP-dependent unconventional secretion pathway. Cell 146, 746-760 (2011).

30. Mizushima, N. Autophagy: process and function. Genes Dev. 21, 2861-2873 (2007).

31. Goldsmith, J., Levine, B. \& Debnath, J. Autophagy and cancer metabolism Methods Enzymol. 542, 25-57 (2014).

32. Nakatogawa, H., Suzuki, K., Kamada, Y. \& Ohsumi, Y. Dynamics and diversity in autophagy mechanisms: lessons from yeast. Nat. Rev. Mol. Cell Biol. 10, 458-467 (2009).

33. Feng, Y., He, D., Yao, Z. \& Klionsky, D. J. The machinery of macroautophagy. Cell Res. 24, 24-41 (2014).

34. Xiong, J. Atg7 in development and disease: panacea or Pandora's Box? Protein Cell 6, 722-734 (2015). 
35. Shintani, T. \& Klionsky, D. J. Autophagy in health and disease: a double-edged sword. Science 306, 990-995 (2004).

36. Cuervo, A. M. Autophagy: in sickness and in health. Trends Cell Biol. 14, 70-77 (2004)

37. Hernández-Gea, V. et al. Autophagy releases lipid that promotes fibrogenesis by activated hepatic stellate cells in mice and in human tissues. Gastroenterology 142, 938-946 (2012).

38. Ding, Y. et al. Autophagy regulates TGF-beta expression and suppresses kidney fibrosis induced by unilateral ureteral obstruction. J. Am. Soc. Nephrology: JASN 25, 2835-2846 (2014).

39. Livingston, M. J. et al. Persistent activation of autophagy in kidney tubular cells promotes renal interstitial fibrosis during unilateral ureteral obstruction. Autophagy 12, 976-998 (2016).

40. Lodder, J. et al. Macrophage autophagy protects against liver fibrosis in mice. Autophagy 11, 1280-1292 (2015).

41. Hidvegi, T. et al. An autophagy-enhancing drug promotes degradation of mutant alpha1-antitrypsin Z and reduces hepatic fibrosis. Science 329, 229-232 (2010).

42. Ghavami, S. et al. Autophagy is a regulator of TGF-beta1-induced fibrogenesis in primary human atrial myofibroblasts. Cell Death Dis. 6, el696 (2015).

43. Gupta, S. S. et al. Inhibition of autophagy inhibits the conversion of cardiac fibroblasts to cardiac myofibroblasts. Oncotarget 7, 78516-78531 (2016).

44. Xue, X., et al. Protein kinase $\mathrm{C}$ alpha drives fibroblast activation and kidney fibrosis by stimulating autophagic flux. J. Biol. Chem. 293, 11119-11130 (2018).

45. $\mathrm{Wu}, \mathrm{J}$. et al. Autophagy promotes fibrosis and apoptosis in the peritoneum during long-term peritoneal dialysis. J. Cell. Mol. Med. 22, 1190-1201 (2018).

46. Pietrocola, F. et al. Regulation of autophagy by stress-responsive transcription factors. Semin Cancer Biol. 23, 310-322 (2013).

47. Wu, T. T., Li, W. M. \& Yao, Y. M. Interactions between autophagy and inhibitory cytokines. Int J. Biol. Sci. 12, 884-897 (2016).

48. Fullgrabe, J., Klionsky, D. J. \& Joseph, B. Histone post-translational modifications regulate autophagy flux and outcome. Autophagy 9, 1621-1623 (2013).

49. Sakamaki, J.-i et al. Bromodomain protein BRD4 is a transcriptional repressor of autophagy and lysosomal function. Mol. Cell 66, 517-532. e519 (2017).

50. Hale, C. M. et al. Identification of modulators of autophagic flux in an imagebased high content siRNA screen. Autophagy 12, 713-726 (2016).

51. Klionsky, D. J. et al. Guidelines for the use and interpretation of assays for monitoring autophagy (3rd edition). Autophagy 12, 1-222 (2016).

52. Kabeya, Y. et al. LC3, a mammalian homologue of yeast Apg8p, is localized in autophagosome membranes after processing. The. EMBO J. 19, 5720-5728 (2000).

53. Fullgrabe, J. et al. The histone H4 lysine 16 acetyltransferase hMOF regulates the outcome of autophagy. Nature 500, 468-471 (2013).

54. Komatsu, M. et al. Impairment of starvation-induced and constitutive autophagy in Atg7-deficient mice. J. Cell Biol. 169, 425-434 (2005).

55. Thoen, L. F. et al. A role for autophagy during hepatic stellate cell activation. J. Hepatol. 55, 1353-1360 (2011).

56. Mallat, A. et al. Autophagy: a multifaceted partner in liver fibrosis. Biomed. Res. Int. 2014, 869390 (2014).

57. Patel, A. S. et al. Autophagy in idiopathic pulmonary fibrosis. PLoS ONE 7, e41394 (2012).

58. Hill, C. et al. Autophagy inhibition-mediated epithelial-mesenchymal transition augments local myofibroblast differentiation in pulmonary fibrosis. Cell Death Dis. 10, 591 (2019).

59. Romero, Y. et al. mTORC1 activation decreases autophagy in aging and idiopathic pulmonary fibrosis and contributes to apoptosis resistance in IPF fibroblasts. Aging Cell 15, 1103-1112 (2016).

60. Cabrera, S. et al. Essential role for the ATG4B protease and autophagy in bleomycin-induced pulmonary fibrosis. Autophagy 11, 670-684 (2015).

61. Sosulski, M. L. et al. Deregulation of selective autophagy during aging and pulmonary fibrosis: the role of TGF $\beta 1$. Aging Cell 14, 774-783 (2015).

62. Kim, M. S. et al. IL-37 attenuates lung fibrosis by inducing autophagy and regulating TGF- $\beta 1$ production in mice. J. Immunol. 203, 2265-2275 (2019).

63. Wang, K. et al. Identification of ANXA2 (annexin A2) as a specific bleomycin target to induce pulmonary fibrosis by impeding TFEB-mediated autophagic flux. Autophagy 14, 269-282 (2018).

64. Araya, J. et al. Insufficient autophagy in idiopathic pulmonary fibrosis. Am. J. Physiol. Lung Cell Mol. Physiol. 304, L56-69 (2013).

65. Mahavadi, P. et al. Regulation of macroautophagy in amiodarone-induced pulmonary fibrosis. J. Pathol. Clin. Res. 1, 252-263 (2015).

66. Krämer, M. et al. Inhibition of H3K27 histone trimethylation activates fibroblasts and induces fibrosis. Ann. Rheum. Dis. 72, 614-620 (2013).

67. Bergmann, C. et al. The histone demethylase Jumonji domain-containing protein 3 (JMJD3) regulates fibroblast activation in systemic sclerosis. Ann. Rheum. Dis. 77, 150-158 (2018).

68. Takahashi, T. et al. Epithelial Flil deficiency drives systemic autoimmunity and fibrosis: Possible roles in scleroderma. J. Exp. Med. 214, 1129-1151 (2017).

69. Ali, S., Nouhi, Z., Chughtai, N. \& Ali, S. SHP-2 regulates SOCS-1-mediated Janus kinase-2 ubiquitination/degradation downstream of the prolactin receptor. J. Biol. Chem. 278, 52021-52031 (2003).
70. Aceto, N. et al. Tyrosine phosphatase SHP2 promotes breast cancer progression and maintains tumor-initiating cells via activation of key transcription factors and a positive feedback signaling loop. Nat. Med. 18, 529-537 (2012).

71. Baroni, S. S. et al. Stimulatory autoantibodies to the PDGF receptor in systemic sclerosis. N. Engl. J. Med. 354, 2667-2676 (2006).

72. van den Hoogen, F. et al. 2013 classification criteria for systemic sclerosis: an American college of rheumatology/European league against rheumatism collaborative initiative. Ann. Rheum. Dis. 72, 1747-1755 (2013).

73. Palumbo-Zerr, K. et al. Orphan nuclear receptor NR4A1 regulates transforming growth factor-beta signaling and fibrosis. Nat. Med. 21, 150-158 (2015).

74. Ran, F. A. et al. Genome engineering using the CRISPR-Cas9 system. Nat. Protoc. 8, 2281-2308 (2013).

75. Beyer, C. et al. Blockade of canonical Wnt signalling ameliorates experimental dermal fibrosis. Ann. Rheum. Dis. 72, 1255-1258 (2013).

76. Dees, C. et al. Platelet-derived serotonin links vascular disease and tissue fibrosis. J. Exp. Med. 208, 961-972 (2011).

77. Chakraborty, D. et al. Activation of STAT3 integrates common profibrotic pathways to promote fibroblast activation and tissue fibrosis. Nat. Commun. 8 , 1130 (2017).

78. Chen, C. W. et al. Pharmacological inhibition of porcupine induces regression of experimental skin fibrosis by targeting Wnt signalling. Ann. Rheum. Dis. 76, 773-778 (2017)

79. Zehender, A. et al. The tyrosine phosphatase SHP2 controls TGFbeta-induced STAT3 signaling to regulate fibroblast activation and fibrosis. Nat. Commun. 9, 3259 (2018).

80. Kaizuka, T. et al. An autophagic flux probe that releases an internal control. Mol. Cell 64, 835-849 (2016).

81. Wolfraim, L. A. et al. Development and application of fully functional epitopetagged forms of transforming growth factor- $\beta$. J. Immunol. Methods 266, 7-18 (2002).

82. Akhmetshina, A. et al. Activation of canonical Wnt signalling is required for TGF-beta-mediated fibrosis. Nat. Commun. 3, 735 (2012).

83. Beyer, C. et al. Stimulation of soluble guanylate cyclase reduces experimental dermal fibrosis. Ann. Rheum. Dis. 71, 1019-1026 (2012).

84. Cox, J. et al. Andromeda: a peptide search engine integrated into the MaxQuant environment. J. Proteome Res. 10, 1794-1805 (2011).

85. Abe, M. et al. An assay for transforming growth factor $-\beta$ using cells transfected with a plasminogen activator inhibitor-1 promoter-luciferase construct. Anal. Biochem. 216, 276-284 (1994).

86. Khan, S. A., Joyce, J. \& Tsuda, T. Quantification of active and total transforming growth factor- $\beta$ levels in serum and solid organ tissues by bioassay. BMC Res. Notes 5, 636 (2012).

87. Sage, D. et al. DeconvolutionLab2: an open-source software for deconvolution microscopy. Methods 115, 28-41 (2017).

88. Dees, C. et al. JAK-2 as a novel mediator of the profibrotic effects of transforming growth factor beta in systemic sclerosis. Arthritis Rheum. 64, 3006-3015 (2012).

89. Distler, A. et al. Inactivation of tankyrases reduces experimental fibrosis by inhibiting canonical Wnt signalling. Ann. Rheum. Dis. 72, 1575-1580 (2013)

90. Zerr, P. et al. Vitamin D receptor regulates TGF-beta signalling in systemic sclerosis. Ann. Rheum. Dis. 74, e20 (2014).

91. Distler, A. et al. Combined inhibition of morphogen pathways demonstrates additive antifibrotic effects and improved tolerability. Ann. Rheum. Dis. 73, 1264-1268 (2014)

92. Ashcroft, T., Simpson, J. M. \& Timbrell, V. Simple method of estimating severity of pulmonary fibrosis on a numerical scale. J. Clin. Pathol. 41, 467-470 (1988).

93. Hubner, R. H. et al. Standardized quantification of pulmonary fibrosis in histological samples. BioTechniques 44, 514-507 (2008). 507-511.

94. Tyanova, S. et al. The Perseus computational platform for comprehensive analysis of (prote) omics data. Nat. Methods 13, 731-740 (2016).

\section{Acknowledgements}

We thank Rita Weinkam, Regina Kleinlein, Lena Summa and Katja Dreißigacker for the excellent technical assistance. We would like to thank the following organizations for funding: Grants DI 1537/7-1, DI 1537/8-1, DI 1537/9-1 and -2, DI 1537/11-1, DI 1537/ 12-1, DI 1537/13-1, DI 1537/14-1, DI 1537/17-1 of the German Research Foundation, SFB CRC1181 (project C01) and SFB TR221/ project number 324392634 (B04) of the German Research Foundation, grants A64 and A79 of the IZKF in Erlangen, grant 2013.056.1 of the Wilhelm-Sander-Foundation, grants 2014_A47 and 2014_A184 of the Else-Kröner-Fresenius-Foundation, grant 14-12-17-1-Bergmann of the ELANFoundation Erlangen, BMBF, MASCARA program, TP 2 and a Career Support Award of Medicine of the Ernst Jung Foundation. Parts of this work were supported by the Deutsche Forschungsgemeinschaft (DFG, German Research Foundation) through CRC829 - 73111208 and RU2722 - 407239409 (to BE) and by the Ministry of Science and Technology, R.O.C through the grant number 106-2320-B-002-004-MY3 (to NYL). 


\section{Author contributions}

Author contributions were as follows: A.Z., N.Y.L., Y.-N.L. and J.H.W.D. designed the study, A.Z., N.Y.L., Y.-N.L., A.S.F., J.N., A.H.G., C.W.C., X.D., J.H., H.Z., L.S., A.S., S.R., C.B., A.R., M.P., and B.E. contributed to acquisition and interpretation of data, A.Z., N.Y.L., Y.-N.L., G.S., and J.H.W.D. prepared the manuscript. All authors read and approved the final version.

\section{Funding}

Open Access funding enabled and organized by Projekt DEAL.

\section{Competing interests}

Although none of the authors has any direct conflict of interest related to autophagy in fibrosis, J.H.W.D. are involved in the development of targeted therapies for fibrotic diseases such as SSc. J.H.W.D. has consultancy relationships with Actelion, Active Biotech, Anamar, ARXX, Bayer Pharma, Boehringer Ingelheim, Celgene, Galapagos, GSK, Inventiva, JB Therapeutics, Medac, Pfizer, RuiYi and UCB. J.H.W.D. has received research funding from Anamar, Active Biotech, Array Biopharma, aTyr, BMS, Bayer Pharma, Boehringer Ingelheim, Celgene, Galapagos, GSK, Inventiva, Novartis, SanofiAventis, RedX, UCB. J.H.W.D. is stock owner of 4D Science and Scientific head of FibroCure. The remaining authors declare no competing interests.

\section{Additional information}

Supplementary information The online version contains supplementary material available at https://doi.org/10.1038/s41467-021-24601-y.
Correspondence and requests for materials should be addressed to J.H.W.D.

Peer review information Nature Communications thanks the anonymous reviewers for their contribution to the peer review of this work.

Reprints and permission information is available at http://www.nature.com/reprints

Publisher's note Springer Nature remains neutral with regard to jurisdictional claims in published maps and institutional affiliations.

(c) (i) Open Access This article is licensed under a Creative Commons Attribution 4.0 International License, which permits use, sharing, adaptation, distribution and reproduction in any medium or format, as long as you give appropriate credit to the original author(s) and the source, provide a link to the Creative Commons license, and indicate if changes were made. The images or other third party material in this article are included in the article's Creative Commons license, unless indicated otherwise in a credit line to the material. If material is not included in the article's Creative Commons license and your intended use is not permitted by statutory regulation or exceeds the permitted use, you will need to obtain permission directly from the copyright holder. To view a copy of this license, visit http://creativecommons.org/ licenses/by/4.0/.

(C) The Author(s) 2021 